\title{
Application Of Fuzzy Logic For User Classification In Personalized Web Search
}

\author{
S. Sendhilkumar ${ }^{1}$, K. Selvakumar ${ }^{2}$ and G.S. Mahalakshmi ${ }^{3}$ \\ ${ }^{1,2,3}$ Dept. of IST, Anna University, Chennai
}

\begin{abstract}
Classifying web users in a personalised search setup is cumbersome due the very nature of dynamism in user browsing history. This fluctuating nature of user behaviour and user interest shall be well interpreted within a fuzzy setting. Prior to analysing user behaviour, nature of user interests has to be collected. This work proposes a fuzzy based user classification model to suit a personalised web search environment. The user browsing data is collected using an established customised browser designed to suit personalisation. The data are fuzzified and fuzzy rules are generated by applying decision trees. Using fuzzy rules, the search pages are labelled to aid grouping of user search interests. Evaluation of the proposed approach proves to be better when compared with Bayesian classifier.
\end{abstract}

\section{KEYWORDS}

Web search, Personalization, Fuzzy classification, Fuzzy rules and World Wide Web

\section{INTRODUCTION}

Web search represents a significant portion of Web activity. There are three basic approaches to web search: i) Search Engines ii) Web Directories and iii) Hyperlinks [1]. However neither of these traditional search processes is effectively made to handle user's interest and their shift in search interest. Therefore results returned by existing search techniques do not provide the acceptable relevant search information. The underlying assumption is that user query is static and the search process has iterated the query recursively until the required information is retrieved $[2,3]$.

Yet, there are difficulties encountered in web search which fall into five categories: i) Dynamic changes in data ii) Difficulties during retrieval of data iii) Understanding the context of search requests iv) Continuous drift in search and v) Difficulties in User and system communication [4,5]. Information on the Web is dynamic, heterogeneous and rapidly increasing, modified, and moved out. It is also un-structured and not self organized, so surfing needed information is dangling in nature [6,7]. In reality, during the search process users learn to go through the information, read the titles in result sets, retrieve the documents themselves, viewing a list of topics related to their query terms and navigating through hyperlinked Web sites [8].

User's information needs are not satisfied by a single search process, there is a mismatch between the retrieved documents and the required information. Information encountered at one search point may lead to a new unanticipated direction, so the user's required information and consequently their queries continually shift. The user model has to translate user requirement into a query form within some agreed upon system model. Keeping track of user's choice made during their search process allows them to return to temporarily unwanted contents, jump from one category of the content to another and, to retain information and the context across search sessions [9-12].

Personalized Web search mechanism effectively obtains the user's real time required information $[13,14]$. Significant research progress exists in this area towards improving search results according to user interest; yet this has not been made into a commercial facility in existing search engines. The reason is that the search results are often interpreted unsatisfactory. 
People usually spend time to browse, read the irrelevant documents and continue to spend their valuable time until they find satisfactory results. The main reason behind this is that it is very hard to understand user's search intention; also it is fuzzy in nature. There is always a continuous change or drift in their search intension. In order to estimate change of interest of the users, following parameters shall be considered during the search process: i) Explicit (denotative) factors like user thrown queries, keywords, URLs, content referred, log-files, link structure, preference of previous users, etc. ii) Implicit (connotative) factors like semantics, term- list, timespent on the page, scrolling speed, size of the page, click-through, maximum scrolling-speed, minimum scrolling-speed, time-spent per page-size, etc. [3,15,16].

The above mentioned user based parameters contains fuzziness due to the dynamism in their interest as well as shifting nature of their intension from one category to another. Collection of these parameters in direct manner is unethical [5]. In order to predict the user interest using these fuzzy nature parameters, personalized search process requires special and effective new user model which incorporates a fuzzy approach [17-19]. This fuzzy model shall be utilised to overcome fuzziness from the various user parameters and accurately predict the user's interest class as well as the interest shift between different user needs. This work proposes a fuzzy based user classification of search results in a personalised web search setting.

The rest of the paper is organized as follows. Section-2 provides the related works of previous Web personalization approaches with soft computing techniques. The proposed user model is described in section-3. Data pre-processes and validation of the user's data set, the relationship between the various users' search parameters, the randomness and uncertainty estimation of the user's data using frequency test and various statistical measures, mathematical notations of various fuzzy membership functions on different user search parameters, generation of fuzzy rules using decision tree algorithm and user grouping are shown in sub sections. Section-4 depicts a discussion and evaluation of results.

\section{RELATED WORK}

Fields like artificial intelligence, web mining and pattern recognition are influenced by many factors. Classification is one such important factor. Heuristic searching techniques forms the basis for majority of common classification algorithms. Finding the subset of the representative rules from the restricted set of training data is the main role of these algorithms [20,21].

Fundamentally there are three objectives: generating user navigation profiles for link prediction; enriching the profiles with semantic information to diversify them, and obtaining global and language-dependent user interest profiles combined with web usage and content mining techniques [22]. In this paper [22], the website content and the standard web usage information (log files from the web server) are taken as input. Semantic structure is automatically extracted and combined with information sources to extract knowledge for many applications. A crispbased approach is used to assign the interest profiles to clusters. User's interests change dynamically during web traversal. Hence it is essential to detect the dynamic web traversal patterns based upon the user's progress. TFPM (Transitional Frequent Pattern Mining) and TUPM (Transitional Utility Pattern Mining) were proposed to detect the web patterns [23].

Analysing students' study environment to suggest personalised activities for each student [24] is another remarkable work in this area. The recommended suggestions were made to reinforce the students' competences in a subject. Characteristics of competences from each subject and the designed activities modelled by fuzzy linguistic labels compute the entire recommendations. A recommendation system based on fuzzy linguistic modelling has this approach as its core. And this approach allows students to receive personalised activities to practice competences which have to reinforce for passing a subject. The unhandled issue is that it is very difficult to determine 
one's affinity with other students because this system is not connected under a social networking platform.

In order to conceptualise documents into concepts using FFCM (Fuzzy Formal Conceptualization Model), a novel classification approach was developed [25, 26]. And this approach reduced the impact from textual ambiguity successfully. This work focuses only on single-class based classification due to the wide-ranging applications of text. In order to provide more complete coverage of FFCM applications, multi-class problems need to be handled. The performance of fuzzy classification systems was improved by reducing the decision subspace and its weight with the help of punishment algorithm [27].

In order to answer user consultations using natural language by Information Extraction (IE) in a set of knowledge platform, a novel method was proposed in [21, 28]. This methodology based on fuzzy logic engine, takes advantage of its flexibility for managing sets of accumulated knowledge. These sets are constructed in a form of a tree structure containing hierarchy levels. Designing and implementing an intelligent agent which can manage any set of knowledge from a space where information is imprecise, vague and abundant is the main goal of this system. Fuzzy logics were also used to summarize text for extracting the most relevant sentences [29].

For assisting the user to find the required experts an expert recommendation was proposed [30]. In order to construct the expert profile, a fuzzy linguistic method was adopted in this method. When the document is registered the fuzzy text classifier is used to get the relevant degree of document to each knowledge area, which forms the base for the construction of user profile. To derive the overall knowledge of the user, the user profile consisting of time and relevance factor of the rated documents is constructed. Based on the similarity between the user profile and the expert profile [31], the expert that fulfils the knowledge needs are recommended. For accurate building of user interest profiles, the method requires the user to rate similar to explicit feedback and the user must be reluctant to rate. But this stands to be a common drawback for all contentbased recommendation methods. In the recommendation process like consulting history and the collaborative filtering, required information will be considered and filtering may be combined to make the recommendation more precise and comprehensive.

Two key technologies, namely pruning the outliers in the training data by SVMs (support vector machines) by eliminating the influence of outliers on the learning process and finding the fuzzy set with sound linguistic interpretation to describe each class based on AFS (Axiomatic Fuzzy Set) theory are fully capitalised by a classification method [32]. Aspects such as the improvement of learning methods in terms of computational complexity, especially in terms of adaptability with large scale data sets have to be improved further.

Although still a prototype, the idea of detecting emotions from the text, in terms of the sentiment polarity and the emotions has been proposed [33]. A fuzzy- based modelling of emotions enables the identification of linguistic patterns that intensify or reduce such emotions. Fuzzy logic is also used for evaluating the normalization completeness [34]. It was carried out in two steps - finding the relation's normal form and scaling the normalization. Even for software effort estimation, fuzzy approaches like Trapezoidal Membership Function (TMF) and Gaussian Membership Function (GMF) can be used as an alternative to traditional approaches like COCOMO model [35]. The use of fuzzy logic provides more flexibility for modelling the emotions; in addition, fuzzy modifiers are an adequate tool to tune these emotions according to the text.

The above mentioned fuzzy system based web mining research are showing their outstanding performance in the uncertain nature of the Web environment. This also proves to be the right decision for the recommendation process to be made from the vague and the uncertain environment in a robust manner. So it concludes that any Web mining application along with fuzzy methodologies will bring accurate estimation of user needs. 


\section{Proposed Work: Fuzzy baSed USer Classification Model}

The proposed user model classifies/groups the users based on their interest labels using fuzzy approach. The classification model has the following components: Data collection and Preprocessing, Fuzzification, Rule generation, Label assignment and Grouping.

The customized Web browser [5] is used for collecting user search data such as: Username, IP address of the system, time spent, scrolling speed, URLs, queries, click through, page size, Scrolling speed and pre-processed using linguistic approaches. The data collected is then fuzzified, decision tree constructed and later rule generation is performed $[3,36]$. The rules are then used to filter the pages that are not relevant to the user's search queries. The selected Web pages and user queries are mapped to categorical labels from ODP taxonomy. Finally the users are grouped based on the categorical labels.

\subsection{Creation of Meta data}

Data for experimental purpose is collected on the client side because various factors that affect personalization are not correctly reflected in the server side. For example page-view time recorded in the server logs is affected by network delay. Also cache hits are not recorded accurately in server logs [3]. In addition, it is very hard and complex to identify the specific user $\log$ information from the server side. Finally scrolling speed cannot be traced from the server side $[3,5]$.

After collecting data from the client side, the search data is pre-processed for extracting various factors that affect personalization. The following useful information is drawn out from the search data such as: i) User queries ii) Web pages visited iii) Scrolling Speed iv) Click through and v) Page size.

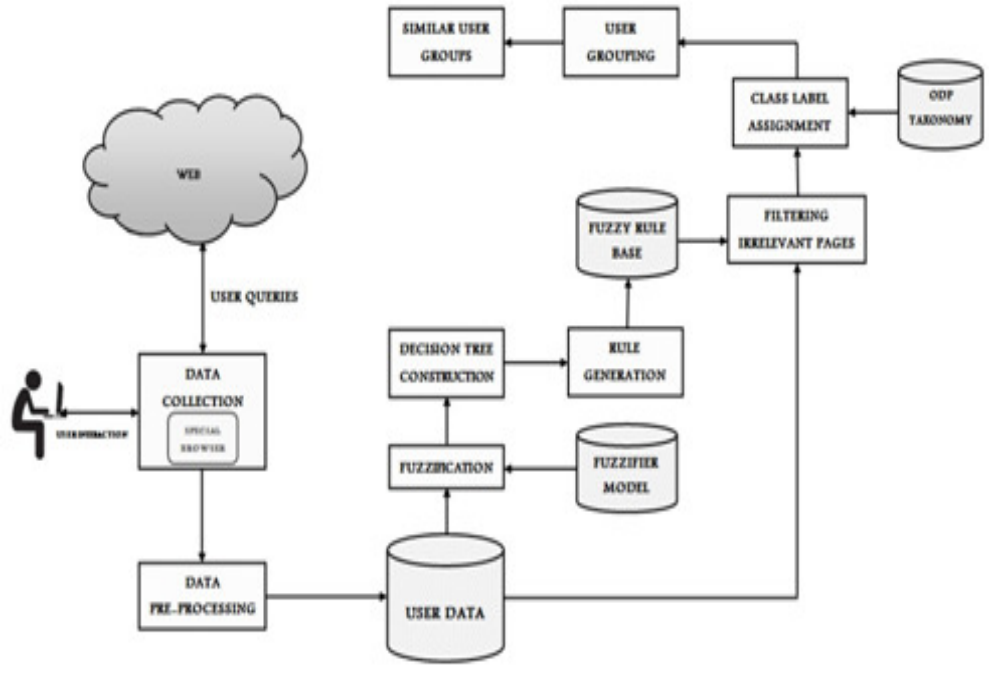

Figure 1. Fuzzy based Classification model

User queries which are the direct indications of user's interest are directly extracted from the input textbox [refer figure 1]. The Web pages visited by the users are parsed. The text content thus extracted is linguistically pre-processed for stop-word removal and stemming. The root words thus identified are used for indicating that Web page in the database. 
Each page visited by the user consists of set of scrolling speeds recorded by the browser [5]. The average, maximum and minimum scrolling speed on a page visited by the user is computed from the set of scrolling speeds. The click through on a page is calculated whenever there was the change in address box in the browser. Change in the content of the address box occurs whenever the user clicks a link/URL available in the page that is currently being visited. The Time/PageSize ratio is derived from the list of pages visited and time spent by the user in the page. A sample raw-data and pre-processed data is shown in Figure 2 \& Figure 3 respectively.

Table 1. Experimental dataset information

\begin{tabular}{|c|l|c|c|}
\hline \multicolumn{5}{|c|}{ User Data Set information } \\
\hline Sl.No & \multicolumn{1}{|c|}{ Factors in user data } & Total & Ratio \\
\hline 1 & Number of Users & 10 & - \\
\hline 2 & Number of search sessions & 79 & 7.9 Search Session/User \\
\hline 3 & Number of search queries & 486 & 48.6 Search Queries/User \\
\hline 4 & Number of Web pages visited & 524 & $\begin{array}{c}52.4 \mathrm{Web} \text { pages } \\
\text { visited/User }\end{array}$ \\
\hline
\end{tabular}

Data collection involved 10 distinct users and their search data (524 pages) visited during their search process. A summarization of the data collected is shown in Table 1. The table also highlights average search sessions, search queries issued and Web pages visited per user.

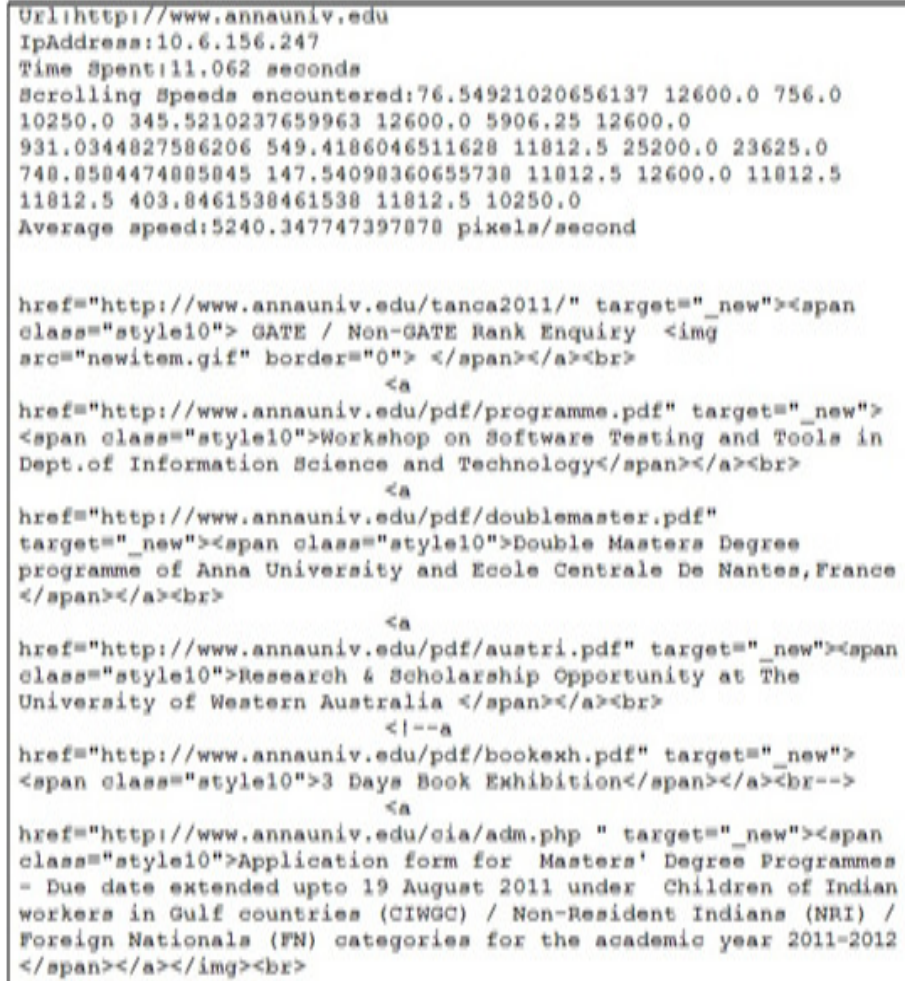

Figure 2: Snapshot representation of sample user data before pre-processing 


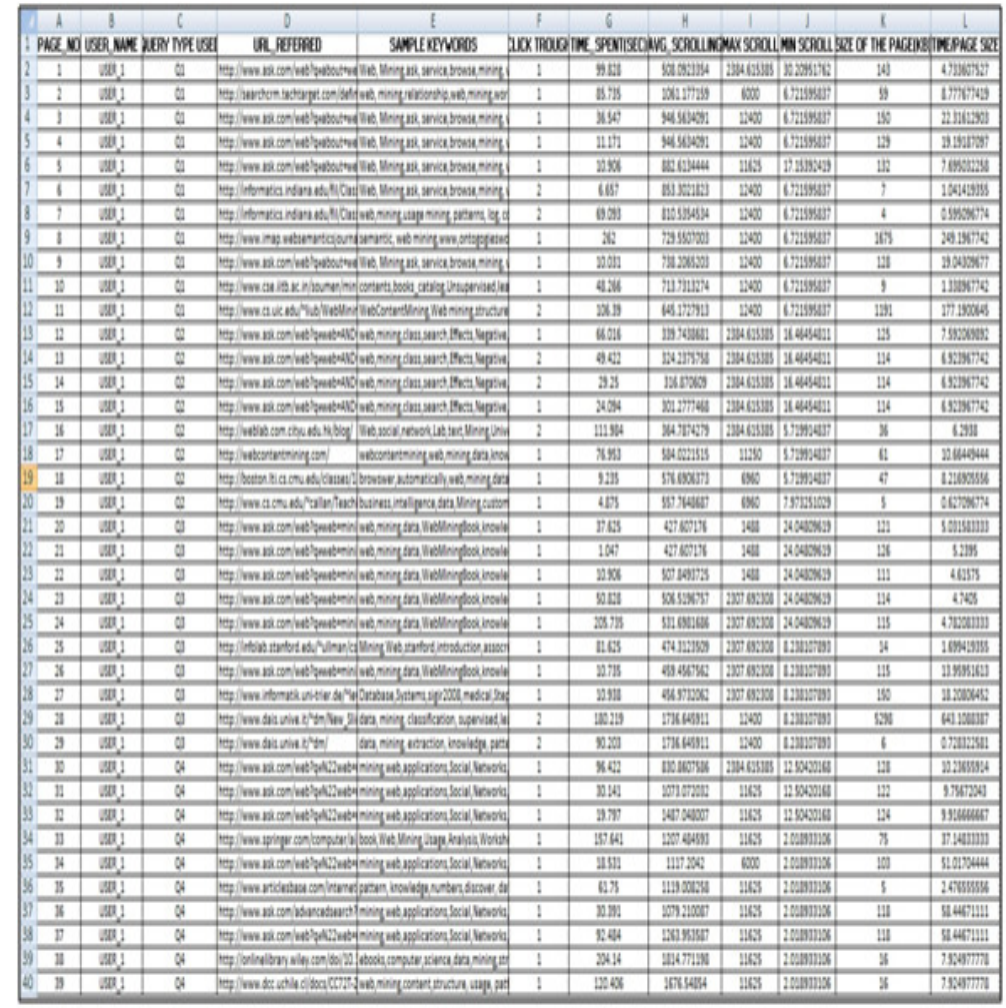

Figure 3: Snapshot representation of sample user data after pre-processing

\subsection{Statistical analysis of pre-processed search data}

The pre-processed user search data are analyzed using SPSS (Statistical Package for the Social Sciences) and StatPlus mathematical tools. The collected user data contains the randomness as well as uncertainty due to drift in their search process. It is tested and measured through frequency test and various statistical measures. A hypothesis test use sample data to test a hypothesis about the population from which the sample was taken. It makes inference about one or more population when sample data are available. Hypothesis test on user data based on timespent on a Web page is considered based on statistical measures (mean, median and mode, etc) such as $\mathrm{H}_{0}$ and $\mathrm{H}_{1}$ called Null hypothesis and Alternative hypothesis respectively. Here $\mathrm{H}_{0}, \mathrm{H}_{1}$ are assumed and represented below

$\mathrm{H}_{0}$ : Data collected highlights randomness as well as uncertainty.

$\mathrm{H}_{1}: \mathrm{H}_{0}$ is not true

The results show that there is an uncertainty that exists in the users' browsing data. In order to resolve these issues fuzzy approach is incorporated with this model to effectively perform interest label based classification on the users. The randomness and uncertainty that exist in the Timespent on a single page visited by the user in various sessions are shown in Table 2. 
Table 2: Randomness and uncertainty existing in Time-spent on single-user's single page visit across various sessions

\begin{tabular}{|c|c|c|}
\hline SESSION & PAGE_NO & TIME_SPENT(Sec) \\
\hline I & $\mathrm{P}_{1}$ & 99.828 \\
\hline & $\mathrm{P}_{1}$ & 85.735 \\
\hline & $\mathrm{P}_{1}$ & 11.171 \\
\hline & $\mathrm{P}_{1}$ & 10.906 \\
\hline II & $\mathrm{P}_{1}$ & 66.016 \\
\hline & $\mathrm{P}_{1}$ & 49.422 \\
\hline & $\mathrm{P}_{1}$ & 29.25 \\
\hline & $\mathrm{P}_{1}$ & 24.094 \\
\hline & $\mathrm{P}_{1}$ & 111.984 \\
\hline III & $\mathrm{P}_{1}$ & 18.531 \\
\hline & $\mathrm{P}_{1}$ & 61.75 \\
\hline & $\mathrm{P}_{1}$ & 30.391 \\
\hline & $\mathrm{P}_{1}$ & 92.484 \\
\hline
\end{tabular}

\subsection{Fuzzification on User search data}

In order to illustrate uncertainty and vagueness mathematically, fuzzy concept is specifically designed. Everything is a matter of degree in fuzzy logic and knowledge is interpreted as a collection of elastic or equally fuzzy constraint on a collection of variables [32-38]. Concept of Linguistic variable plays an important role in fuzzy logic and their values are words or sentences in natural language $[39,40]$. Any relation between two linguistic variables can be expressed in terms of fuzzy if-then rules [41, 42].

Fuzzy sets have been applied in many fields [18] in which uncertainty plays a key role. In particular, Web search area is an excellent example of vagueness and uncertainty [19, 43, 44]. Here the user interest is uncertain and fuzzy in nature; it is not simply classified using binary class labels (like Yes or No). In order to resolve this fuzziness in user interest, the pages visited by users are classified under labels like Not-Interested, Weakly-Interested, Mediumly-Interested and Strongly-Interested.

\subsubsection{Fuzzification}

The process of transforming crisp values into grades of membership for linguistic terms of fuzzy sets is known as fuzzification [25-26, 28, 45-47]. In this work, linguistic variables are evaluated using both triangular and trapezoidal membership functions and accompanied by degree of membership ranging from 0 to 1 . The user data and its distribution is compared with various fuzzification membership functions. The collected user data like time-spent and scrolling-speed resembles triangular and trapezoidal membership functions respectively. Therefore this model utilizes both Triangular and Trapezoidal fuzzifiers for the fuzzification of user data as shown in Eq. $1 \& 3$. Fuzzification of given user data can be performed by opting input parameters into Xaxis (Horizontal line) and representing Y-axis (Vertical line) with the upper limit of the membership function for estimating the degree of membership.

\subsubsection{Fuzzification of time-spent using Triangular Fuzzy membership function}

Triangular function is defined by a lower limit $a$, an upper limit $b$, a value $c$, and $x$ is current time-spent value, where $a<b<c$. It is a fuzzy number represented by three points as follows: 


$$
\mu_{\text {Tines_Spent }}(x, a, b, c)= \begin{cases}0 & x<a \\ \frac{x-a}{b-a} & a \leq x \leq b \\ \frac{c-x}{c-b} & b \leq x \leq c \\ 0 & c \leq z\end{cases}
$$

Here the entire user time-spent values are divided into three bins such as $B_{1}, B_{2}$ and $B_{3}$ and their mean values are calculated and assigned to $a, b, c$ respectively The Triangular fuzzifier is applied on Time-Spent value of the user and the boundary values are defined based on bin-mean approach. The number of bin values is chosen based on the number of boundary values of the specific fuzzifier function.

For example, for 524 Web pages visited by an individual, the time-spent value is sorted and divided into three bins of sizes 174, 174 and 176 each. Here the first bin $\mathrm{B}_{1}$ values and its mean value is represented. The below bin $B_{1}$ contains 174 time-spent values in sorted form, and its mean value is calculated as $\mathbf{1 6 . 4 3 6 3 1}$. The boundary values of this fuzzifier are shown in the Figure 4.

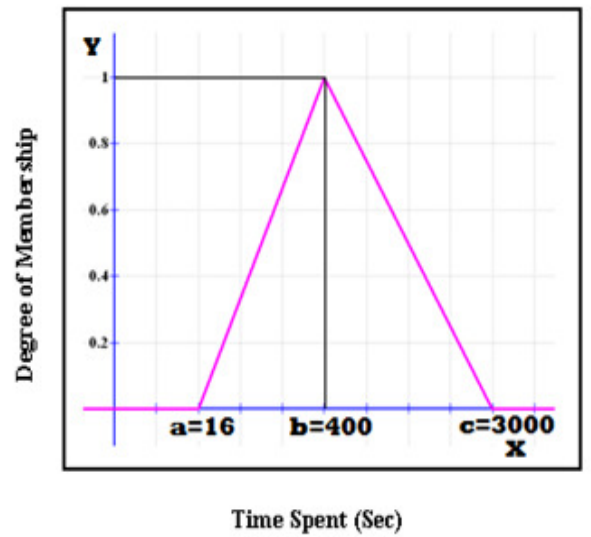

Figure 4: Boundary values of Triangular fuzzifier 
$\mathrm{B}_{1}=[1.016,1.031,1.047,1.094,1.125,1.406,2.031,2.485,3.485,4.844,4.875,5.203,5.563$, 5.719, 5.781, 6.297, 6.657, 7.125, 7.234, 7.375, 7.391, 7.516, 7.531, 7.594, 8.125, 8.453, 8.625, $8.641,8.781,8.859,8.984,9.235,9.39,9.953,9.969,10.031,10.032,10.047,10.062,10.11$, $10.375,10.39,10.391,10.641,10.656,10.735,10.781,10.844,10.906,10.906,10.907,10.938$, 10.985, 11.031, 11.063, 11.094, 11.094, 11.125, 11.125, 11.125, 11.171, 11.203, 11.297, 11.437, $11.438,11.625,12.062,12.062,12.063,12.109,12.188,12.312,12.328,12.375,12.891,13$, $13.203,13.328,13.375,13.5,13.718,13.719,13.734,13.75,13.906,13.953,14.125,14.188$, $14.484,14.734,14.734,14.844,14.953,15.469,15.578,16.234,16.312,16.359,16.406,16.515$, $16.64,17.516,17.516,17.813,18.016,18.531,19.5,19.594,19.703,19.797,19.875,20.265$, $20.578,20.687,20.71,21.047,21.188,21.203,21.218,21.297,21.922,21.984,22.046,22.079$, $22.188,22.281,22.625,22.625,22.641,23.313,23.344,23.562,23.875,23.907,24.094,24.141$, $24.39,24.531,24.563,25.094,25.438,25.484,25.531,25.75,26.031,26.141,26.437,26.625$, $26.671,26.797,27.329,27.969,28.093,28.344,28.359,28.859,28.875,29.156,29.25,29.984$, $30.015,30.141,30.25,30.296,30.797,30.844,30.984,31.125,31.265,31.281,31.5,31.687$, $31.75,32.438]$

$$
\begin{gathered}
B_{1} \text { Mean }=\frac{\sum_{i=1}^{N} \text { timpe }- \text { spent }_{i}}{N} \\
B_{1} \text { mean }=16.43631
\end{gathered}
$$

Table 3: Time-spent parameter equivalent to Triangular fuzzy function based membership values

\begin{tabular}{|c|c|c|}
\hline Page No. & Time-Spent(Sec) & Triangular Fuzzifier \\
\hline $\mathrm{P}_{1}$ & 99.828 & 0.028092 \\
\hline $\mathrm{P}_{2}$ & 85.735 & 0.02337 \\
\hline $\mathrm{P}_{3}$ & 36.547 & 0.006886 \\
\hline $\mathrm{P}_{4}$ & 11.171 & 0 \\
\hline $\mathrm{P}_{5}$ & 10.906 & 0 \\
\hline $\mathrm{P}_{6}$ & 6.657 & 0 \\
\hline $\mathrm{P}_{7}$ & 69.093 & 0.017793 \\
\hline $\mathrm{P}_{8}$ & 262 & 0.08244 \\
\hline $\mathrm{P}_{9}$ & 10.031 & 0 \\
\hline $\mathrm{P}_{10}$ & 48.266 & 0.010813 \\
\hline $\mathrm{P}_{11}$ & 106.39 & 0.030292 \\
\hline $\mathrm{P}_{12}$ & 66.016 & 0.016761 \\
\hline $\mathrm{P}_{13}$ & 49.422 & 0.0112 \\
\hline $\mathrm{P}_{14}$ & 29.25 & $4.44 \mathrm{E}-03$ \\
\hline $\mathrm{P}_{15}$ & 24.094 & 0.002712 \\
\hline $\mathrm{P}_{16}$ & 111.984 & 0.032166 \\
\hline $\mathrm{P}_{17}$ & 76.953 & 0.020427 \\
\hline $\mathrm{P}_{18}$ & 9.235 & 0 \\
\hline $\mathrm{P}_{19}$ & 4.875 & 0 \\
\hline $\mathrm{P}_{20}$ & 37.625 & 0.007246984 \\
\hline
\end{tabular}


Similarly other two bin-mean values $\mathrm{B}_{2}$ and $\mathrm{B}_{3}$ are estimated and assigned to the corresponding boundary values $\boldsymbol{b}$ and $\boldsymbol{c}$ respectively. The remaining parameters such as page-size and time/pagesize are approached in the same method and the membership values of these personalization parameters are derived. Time-spent parameter equivalent to Triangular fuzzy function based membership values for twenty Web pages visited (Pages $\mathrm{P}_{1}-\mathrm{P}_{20}$ ) by a single user during his/her Web search is shown in Table 3.

\subsubsection{Fuzzification of time-spent using Trapezoidal Fuzzy membership function}

A trapezoidal function is defined by a lower limit $a$, an upper limit $d$, a lower support limit $b$, an upper support limit $c$ and current scrolling-speed $x$, where $a<b<c<d$. It is specified by four parameters $a, b, c, d$ follows:

$$
\mu_{\text {Scrolinns-Speed } d}(x, a, b, c, d)= \begin{cases}0 & x<a \\ \frac{(x-a)}{(b-a)} & a \leq x \leq b \\ 1 & b \leq x \leq c \\ \frac{(d-x)}{(d-c)} & c \leq x \leq d \\ 0 & d<x\end{cases}
$$

The Trapezoidal fuzzifier is applied on Scrolling-Speed of the user parameter and its boundary values are initialized to $a=100, b=1000, c=2000$ and $d=3000$ using bin-mean approach. In this case boundary values are four therefore the entire user dataset is divided into four bins and its mean values are estimated accordingly. Initialization of boundary values of this fuzzifier is shown in Figure 5. Table 4 highlights the fuzzified scrolling speed collected on a sample of $20 \mathrm{Web}$ pages (Pages $\left.\mathrm{P}_{1}-\mathrm{P}_{20}\right)$ visited by an individual user.

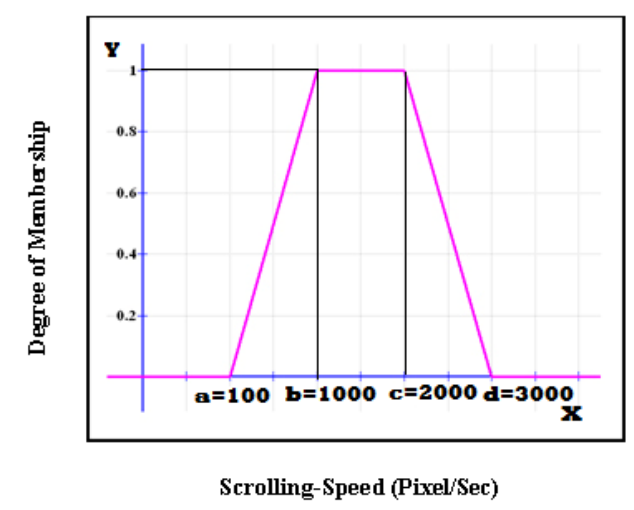

Figure 5. Boundary values of Trapezoidal fuzzifier 
Table 4. Scrolling-speed parameter equivalent to Trapezoidal fuzzy function based membership values

\begin{tabular}{|c|c|c|}
\hline Page No. & $\begin{array}{c}\text { Scrolling- } \\
\text { Speed } \\
\text { (Pixel/Sec) }\end{array}$ & $\begin{array}{c}\text { Trapezoidal } \\
\text { Fuzzifier }\end{array}$ \\
\hline $\mathrm{P}_{1}$ & 508.0923354 & 0.78521456 \\
\hline $\mathrm{P}_{2}$ & 1061.177159 & 0.494117285 \\
\hline $\mathrm{P}_{3}$ & 946.5634091 & 0.554440311 \\
\hline $\mathrm{P}_{4}$ & 946.5634091 & 0.554440311 \\
\hline $\mathrm{P}_{5}$ & 882.6134444 & 0.588098187 \\
\hline $\mathrm{P}_{6}$ & 853.3021823 & 0.603525167 \\
\hline $\mathrm{P}_{7}$ & 810.5354534 & 0.626033972 \\
\hline $\mathrm{P}_{8}$ & 729.5507003 & 0.668657526 \\
\hline $\mathrm{P}_{9}$ & 738.2065203 & 0.664101831 \\
\hline $\mathrm{P}_{10}$ & 713.7313274 & 0.676983512 \\
\hline $\mathrm{P}_{11}$ & 645.1727913 & 0.713066952 \\
\hline $\mathrm{P}_{12}$ & 339.7438681 & 0.873819017 \\
\hline $\mathrm{P}_{13}$ & 324.2375758 & 0.881980223 \\
\hline $\mathrm{P}_{14}$ & 316.870609 & 0.885857574 \\
\hline $\mathrm{P}_{15}$ & 301.2777468 & 0.894064344 \\
\hline $\mathrm{P}_{16}$ & 364.7874279 & 0.860638196 \\
\hline $\mathrm{P}_{17}$ & 584.0221515 & 0.745251499 \\
\hline $\mathrm{P}_{18}$ & 576.6906373 & 0.749110191 \\
\hline $\mathrm{P}_{19}$ & 557.7648687 & 0.759071122 \\
\hline $\mathrm{P}_{20}$ & 427.607176 & 0.827575171 \\
\hline
\end{tabular}

\subsection{Fuzzy Rule Generation}

In this paper, the linguistic variable values which are used for representing various input personalized parameters are low, low-medium and medium and high. Not-interested [NI], Weakly-interested [WI], Mediumly-interested [MI] and Strongly-interested [SI] are the output parameters respectively. A decision tree technique is used to generate fuzzy if-then rules for classifying the user interest. Before generating fuzzy rules the entire personalized user parameters are fuzzified and assigned linguistic labels based on their fuzzy membership values discussed from the previous section 3.3.

The fuzzified values for $20 \mathrm{Web}$ pages visited by an individual are given in Table 6 . The fuzzified values and its equivalent linguistic labels are represented in the Tables 7. Here LMD, MED, HGH labels represents Low-medium, Medium and High respectively. 
International Journal on Cybernetics \& Informatics (IJCI) Vol. 3, No. 3, June 2014

Table 5: Normalized information about the various user parameters

\begin{tabular}{|c|c|c|c|c|}
\hline SL.NO & ATTRIBUTE & $\begin{array}{c}\text { NORMALIZED } \\
\text { VALUE }\end{array}$ & $\begin{array}{l}\text { LINGUISTIC } \\
\text { LABELS }\end{array}$ & $\begin{array}{c}\text { FUZZIFIER } \\
\text { APPROACHED }\end{array}$ \\
\hline 1 & Time Spent & $\begin{array}{l}<16 \\
16-400 \\
400-3000 \\
>3000\end{array}$ & $\begin{array}{l}\text { Low [1] } \\
\text { Low Medium } \\
{[2]} \\
\text { Medium [3] } \\
\text { High [4] }\end{array}$ & Triangular \\
\hline 2 & $\begin{array}{l}\text { Scrolling } \\
\text { Speed }\end{array}$ & $\begin{array}{l}<100 \\
100-1000 \\
1000-2000 \\
>2000\end{array}$ & $\begin{array}{l}\text { Low [1] } \\
\text { Low Medium } \\
{[2]} \\
\text { Medium [3] } \\
\text { High [4] }\end{array}$ & Trapezoidal \\
\hline 3 & Click Through & $\begin{array}{l}<1 \\
1-4 \\
>4\end{array}$ & $\begin{array}{l}\text { Low [1] } \\
\text { Medium [2] } \\
\text { High [3] }\end{array}$ & Trapezoidal \\
\hline 4 & Page Size & $\begin{array}{l}<10 \\
10-105 \\
105-203 \\
>203\end{array}$ & $\begin{array}{l}\text { Low [1] } \\
\text { Low Medium } \\
{[2]} \\
\text { Medium [3] } \\
\text { High [4] }\end{array}$ & Triangular \\
\hline 5 & Max Scrolling & $\begin{array}{l}<1936 \\
1936-8582 \\
8582-15790 \\
>15790\end{array}$ & $\begin{array}{l}\text { Low [1] } \\
\text { Low Medium } \\
{[2]} \\
\text { Medium [3] } \\
\text { High [4] }\end{array}$ & Trapezoidal \\
\hline 6 & Min Scrolling & $\begin{array}{l}<4.5 \\
4.5-30.20 \\
>30.20\end{array}$ & $\begin{array}{l}\text { Low [1] } \\
\text { Medium [2] } \\
\text { High [3] }\end{array}$ & Trapezoidal \\
\hline 7 & $\begin{array}{l}\text { Time } \\
\text { Spent/Page } \\
\text { Size }\end{array}$ & $\begin{array}{l}<0.098 \\
0.098-12.27 \\
12.27-19.05 \\
>19.05\end{array}$ & $\begin{array}{l}\text { Low [1] } \\
\text { Low Medium } \\
{[2]} \\
\text { Medium [3] } \\
\text { High [4] }\end{array}$ & Triangular \\
\hline 8 & $\begin{array}{l}\text { Type of } \\
\text { Interest (Class } \\
\text { Label) }\end{array}$ & ----- & $\begin{array}{l}\text { Not-Interested } \\
\text { [1] } \\
\text { Weakly- } \\
\text { Interested [2] } \\
\text { Mediumly- } \\
\text { Interested [3] } \\
\text { Strongly- } \\
\text { Interested [4] }\end{array}$ & ---- \\
\hline
\end{tabular}

\subsubsection{Fuzzy Decision tree based Fuzzy Rule Generation}

Proposed work analyzes some possible variants of making classification rules from a fuzzy decision tree based on cumulative information. Decision trees, which make use of fuzzy sets and fuzzy logic for solving the introduced uncertainties, are called Fuzzy decision trees (FDTs) [4850]. Fuzzy decision trees mixes part of symbolic and sub-symbolic approaches. Fuzzy sets and symbolic logic permit modeling language-related uncertainties: whereas providing a symbolic framework for data quality. This work projected a brand new interpretation of Fuzzy C4.5, which relies on accumulative data estimate. 
International Journal on Cybernetics \& Informatics (IJCI) Vol. 3, No. 3, June 2014

Table 6: Fuzzified user data

\begin{tabular}{|c|c|c|c|c|c|c|c|}
\hline $\begin{array}{c}\text { Page } \\
\text { no. }\end{array}$ & $\begin{array}{c}\text { Time } \\
\text { spent }\end{array}$ & $\begin{array}{c}\text { Avg } \\
\text { scroll }\end{array}$ & $\begin{array}{c}\text { Click } \\
\text { through }\end{array}$ & $\begin{array}{c}\text { Page } \\
\text { size }\end{array}$ & $\begin{array}{c}\text { Max } \\
\text { scroll }\end{array}$ & $\begin{array}{c}\text { Min } \\
\text { scroll }\end{array}$ & $\begin{array}{c}\text { Time/page } \\
\text { size ratio }\end{array}$ \\
\hline $\mathrm{P}_{1}$ & 2 & 1 & 1 & 1 & 1 & 1 & 2 \\
\hline $\mathrm{P}_{2}$ & 2 & 2 & 2 & 1 & 3 & 2 & 3 \\
\hline $\mathrm{P}_{3}$ & 2 & 3 & 2 & 3 & 3 & 1 & 2 \\
\hline $\mathrm{P}_{4}$ & 2 & 4 & 2 & 2 & 3 & 1 & 4 \\
\hline $\mathrm{P}_{5}$ & 1 & 1 & 1 & 1 & 1 & 1 & 4 \\
\hline $\mathrm{P}_{6}$ & 1 & 1 & 1 & 1 & 1 & 1 & 4 \\
\hline $\mathrm{P}_{7}$ & 4 & 3 & 1 & 4 & 4 & 1 & 2 \\
\hline $\mathrm{P}_{8}$ & 3 & 1 & 1 & 1 & 1 & 1 & 3 \\
\hline $\mathrm{P}_{9}$ & 1 & 1 & 1 & 1 & 1 & 1 & 4 \\
\hline $\mathrm{P}_{10}$ & 2 & 1 & 1 & 1 & 1 & 1 & 2 \\
\hline $\mathrm{P}_{11}$ & 3 & 2 & 2 & 4 & 3 & 2 & 2 \\
\hline $\mathrm{P}_{12}$ & 4 & 3 & 1 & 4 & 4 & 1 & 2 \\
\hline $\mathrm{P}_{13}$ & 2 & 1 & 1 & 1 & 1 & 1 & 2 \\
\hline $\mathrm{P}_{14}$ & 2 & 1 & 1 & 1 & 1 & 1 & 2 \\
\hline $\mathrm{P}_{15}$ & 4 & 1 & 1 & 1 & 1 & 1 & 4 \\
\hline $\mathrm{P}_{16}$ & 3 & 2 & 2 & 3 & 3 & 2 & 1 \\
\hline $\mathrm{P}_{17}$ & 2 & 3 & 1 & 1 & 3 & 2 & 3 \\
\hline $\mathrm{P}_{18}$ & 2 & 3 & 1 & 1 & 3 & 1 & 1 \\
\hline $\mathrm{P}_{19}$ & 1 & 1 & 1 & 1 & 1 & 1 & 4 \\
\hline $\mathrm{P}_{20}$ & 2 & 1 & 1 & 1 & 1 & 1 & 2 \\
\hline
\end{tabular}

Table 7. Linguistic labels based user data with feedback

\begin{tabular}{|c|c|c|c|c|c|c|c|c|}
\hline $\begin{array}{l}\text { Page } \\
\text { no. }\end{array}$ & $\begin{array}{l}\text { Time } \\
\text { spent }\end{array}$ & $\begin{array}{l}\text { Avg } \\
\text { scroll }\end{array}$ & $\begin{array}{c}\text { Click } \\
\text { through }\end{array}$ & $\begin{array}{c}\text { Page } \\
\text { size }\end{array}$ & $\begin{array}{l}\text { Max } \\
\text { scroll }\end{array}$ & $\begin{array}{l}\text { Min } \\
\text { scroll }\end{array}$ & $\begin{array}{l}\text { Time page } \\
\text { size ratio }\end{array}$ & $\begin{array}{l}\text { Feedback } \\
\text { class label }\end{array}$ \\
\hline $\mathrm{P}_{1}$ & LMD & Low & Low & Low & Low & LOW & LMD & wI \\
\hline $\mathrm{P}_{2}$ & LMD & LMD & MED & Low & MED & MED & MED & wI \\
\hline $\mathrm{P}_{3}$ & LMD & MED & MED & MED & MED & Low & LMD & WI \\
\hline $\mathrm{P}_{4}$ & LMD & $\mathrm{HGH}$ & MED & LMD & MED & Low & $\mathrm{HGH}$ & NI \\
\hline $\mathrm{P}_{5}$ & Low & Low & Low & Low & Low & Low & $\mathrm{HGH}$ & NI \\
\hline $\mathrm{P}_{6}$ & Low & Low & Low & Low & LOW & Low & $\mathrm{HGH}$ & NI \\
\hline $\mathrm{P}_{7}$ & HGH & MED & LOW & HGH & $\mathrm{HGH}$ & LOW & LMD & wI \\
\hline $\mathrm{P}_{8}$ & MED & Low & Low & Low & Low & Low & MED & MII \\
\hline $\mathrm{P}_{9}$ & Low & Low & LOW & Low & LOW & Low & $\mathrm{HGH}$ & NI \\
\hline $\mathrm{P}_{10}$ & LMID & Low & Low & Low & Low & Low & LMD & WI \\
\hline $\mathrm{P}_{11}$ & MED & LMD & MED & $\mathrm{HGH}$ & MED & MED & LMD & MII \\
\hline $\mathrm{P}_{12}$ & HGH & MED & LOW & HGH & $\mathrm{HGH}$ & Low & LMD & MII \\
\hline $\mathrm{P}_{13}$ & LMD & Low & Low & Low & Low & Low & LMD & wI \\
\hline $\mathrm{P}_{14}$ & LMD & Low & LOW & LOW & LOW & Low & LMD & wI \\
\hline $\mathrm{P}_{15}$ & $\mathrm{HGH}$ & Low & Low & Low & Low & Low & $\mathrm{HGH}$ & wI \\
\hline $\mathrm{P}_{16}$ & MED & LMD & MED & MED & MED & MED & LOW & MII \\
\hline $\mathrm{P}_{17}$ & LMD & MED & Low & Low & MED & MED & MED & WI \\
\hline $\mathrm{P}_{18}$ & LMD & MED & LOW & Low & MED & Low & LOW & NI \\
\hline $\mathrm{P}_{19}$ & Low & Low & LOW & Low & LOW & Low & $\mathrm{HGH}$ & NI \\
\hline $\mathrm{P}_{20}$ & LMD & Low & LOW & Low & Low & Low & LMD & wI \\
\hline
\end{tabular}

C4.5 is a propagation of ID3 that improves computing potency, deals with continuous values, handles attributes with missing values, avoids over fitting, and performs different functions [5154]. Fuzzified $524 \mathrm{Web}$ pages of user data is provided as an input to C4.5 algorithm. Both testing 
and training data sets are divided using bootstrap approach in order to generate the accurate decision tree. The decision tree thus constructed is given in figure 6 .

User given feedback label based sample training dataset is shown in Table 7.This approach correctly classified 497 instances out of 524 and incorrectly classified instances are 27 and also its mean absolute error, root mean squared error and relative absolute error, etc are shown in Table 8.

Table 8: Various measures conducted on $\mathrm{C} 4.5$ decision tree generation process

\begin{tabular}{|c|l|c|}
\hline Sl.No & \multicolumn{1}{|c|}{ Various Measures } & Percentage \\
\hline 1 & Correctly Classified Instances & $94.8473 \%$ \\
\hline 2 & $\begin{array}{l}\text { Incorrectly Classified } \\
\text { Instances }\end{array}$ & $5.1526 \%$ \\
\hline 3 & Mean absolute error & $0.0462 \%$ \\
\hline 4 & Root mean absolute error & $0.152 \%$ \\
\hline 5 & Relative absolute error & $14.5805 \%$ \\
\hline 6 & Root Relative absolute error & $38.413 \%$ \\
\hline 7 & $\begin{array}{l}\text { Coverage of cases (0.95 } \\
\text { level) }\end{array}$ & $100 \%$ \\
\hline 8 & Total Number of Instances & 524 \\
\hline
\end{tabular}

\subsubsection{Fuzzy classification rule induction}

In this empirical research work, we applied the above procedure on the various user attributes and generate 25 rules. The set of sample rules are shown in the Table 9. The same rules are applied for 10 different users in order for checking its completeness and consistency. Each user's interest may vary according to their different and depends on several factors. This user model considers the user interest as the decision variable.

The attributes that are playing major role in the user interest classification and the notations that are used in this work is normalized and presented in Table 9 also C4.5 algorithm generated tree equivalent rules are represented in Figure 7.

Table 9. Fuzzy based rules for classification of users

\begin{tabular}{|c|c|c|c|c|c|c|}
\hline $\begin{array}{c}\text { Rule } \\
\text { no }\end{array}$ & $\begin{array}{c}\text { Time } \\
\text { spent }\end{array}$ & $\begin{array}{c}\text { Avg } \\
\text { scroll }\end{array}$ & $\begin{array}{c}\text { Click } \\
\text { through }\end{array}$ & $\begin{array}{c}\text { Page } \\
\text { size }\end{array}$ & $\begin{array}{c}\text { Time/page } \\
\text { size ratio }\end{array}$ & Interest \\
\hline 1 & Low & Low & Low & Low & High & $\begin{array}{c}\text { NOT } \\
\text { INTEREST }\end{array}$ \\
\hline 2 & $\begin{array}{c}\text { Low } \\
\text { Medium }\end{array}$ & Low & Low & Low & $\begin{array}{c}\text { Low } \\
\text { Medium }\end{array}$ & $\begin{array}{c}\text { WEAKLY } \\
\text { INTEREST }\end{array}$ \\
\hline 3 & Medium & Low & Low & Low & Medium & $\begin{array}{c}\text { MEDIUM } \\
\text { INTEREST }\end{array}$ \\
\hline 4 & High & Low & Low & Low & High & $\begin{array}{c}\text { STRONGLY } \\
\text { INTEREST }\end{array}$ \\
\hline 5 & $\begin{array}{c}\text { Low } \\
\text { Medium }\end{array}$ & Medium & Medium & Medium & $\begin{array}{c}\text { Low } \\
\text { Medium }\end{array}$ & $\begin{array}{c}\text { WEAKLY } \\
\text { INTEREST }\end{array}$ \\
\hline 7 & Medium & $\begin{array}{c}\text { Low } \\
\text { Hedium }\end{array}$ & Medium & High & $\begin{array}{c}\text { Low } \\
\text { Medium }\end{array}$ & $\begin{array}{c}\text { MEDIUM } \\
\text { INTEREST }\end{array}$ \\
\hline 8 & Medium & High & Medium & How & Medium & $\begin{array}{c}\text { Medium } \\
\text { INEAKLY } \\
\text { INTEREST }\end{array}$ \\
\hline
\end{tabular}


International Journal on Cybernetics \& Informatics (IJCI) Vol. 3, No. 3, June 2014

\begin{tabular}{|c|c|c|c|c|c|c|}
\hline 9 & Medium & Medium & High & Medium & High & $\begin{array}{c}\text { WEAKLY } \\
\text { INTEREST }\end{array}$ \\
\hline 10 & $\begin{array}{c}\text { Low } \\
\text { Medium }\end{array}$ & High & Medium & $\begin{array}{c}\text { Low } \\
\text { Medium }\end{array}$ & High & $\begin{array}{c}\text { NOT } \\
\text { INTEREST }\end{array}$ \\
\hline 11 & High & $\begin{array}{c}\text { Low } \\
\text { Medium }\end{array}$ & High & High & Medium & $\begin{array}{c}\text { STRONGLY } \\
\text { INTEREST }\end{array}$ \\
\hline 12 & $\begin{array}{c}\text { Low } \\
\text { Medium }\end{array}$ & $\begin{array}{c}\text { Low } \\
\text { Medium }\end{array}$ & Medium & Low & Medium & $\begin{array}{c}\text { WEAKLY } \\
\text { INTEREST }\end{array}$ \\
\hline 13 & Medium & $\begin{array}{c}\text { Low } \\
\text { Medium }\end{array}$ & Medium & Medium & Low & $\begin{array}{c}\text { WEAKLY } \\
\text { INTEREST }\end{array}$ \\
\hline 14 & High & $\begin{array}{c}\text { Low } \\
\text { Medium }\end{array}$ & High & Medium & Medium & $\begin{array}{c}\text { MEDIUM } \\
\text { INTEREST }\end{array}$ \\
\hline 15 & Medium & Low & High & High & $\begin{array}{c}\text { Low } \\
\text { Medium }\end{array}$ & $\begin{array}{c}\text { WEAKLY } \\
\text { INTEREST }\end{array}$ \\
\hline 16 & $\begin{array}{c}\text { Low } \\
\text { Medium }\end{array}$ & Medium & Low & Low & Low & $\begin{array}{c}\text { NOT } \\
\text { INTEREST }\end{array}$ \\
\hline 17 & High & $\begin{array}{c}\text { Low } \\
\text { Medium }\end{array}$ & Low & Low & Medium & $\begin{array}{c}\text { MEDIUM } \\
\text { INTEREST }\end{array}$ \\
\hline 18 & $\begin{array}{c}\text { Low } \\
\text { Medium }\end{array}$ & Medium & Low & Low & Medium & $\begin{array}{c}\text { NOT } \\
\text { INTEREST }\end{array}$ \\
\hline 19 & High & Medium & Medium & Medium & Medium & $\begin{array}{c}\text { MEDIUM } \\
\text { INTEREST }\end{array}$ \\
\hline 20 & High & Medium & Low & High & $\begin{array}{c}\text { Low } \\
\text { Medium }\end{array}$ & $\begin{array}{c}\text { WEAKLY } \\
\text { INTEREST }\end{array}$ \\
\hline
\end{tabular}

\subsection{User Query mapping to ODP}

The proposed user model then categorizes the user referred Web with the help of ODP taxonomy. Table 10 contains different query types of the single user in three sessions mapped to keyword based ODP taxonomical categories. Here the category estimation is performed based on a frequency count of each keyword from the user query which is compared with ODP taxonomy. It represents the number of times that particular keyword is matched with the entire list of the ODP structure. In each session there are five different forms of queries like keyword query, Boolean query, phrase query, proximity query and natural language query and the same are represented by $\mathrm{Q}_{1}, \mathrm{Q}_{2}, \mathrm{Q}_{3}, \mathrm{Q}_{4}$, and $\mathrm{Q}_{5}$ respectively. 


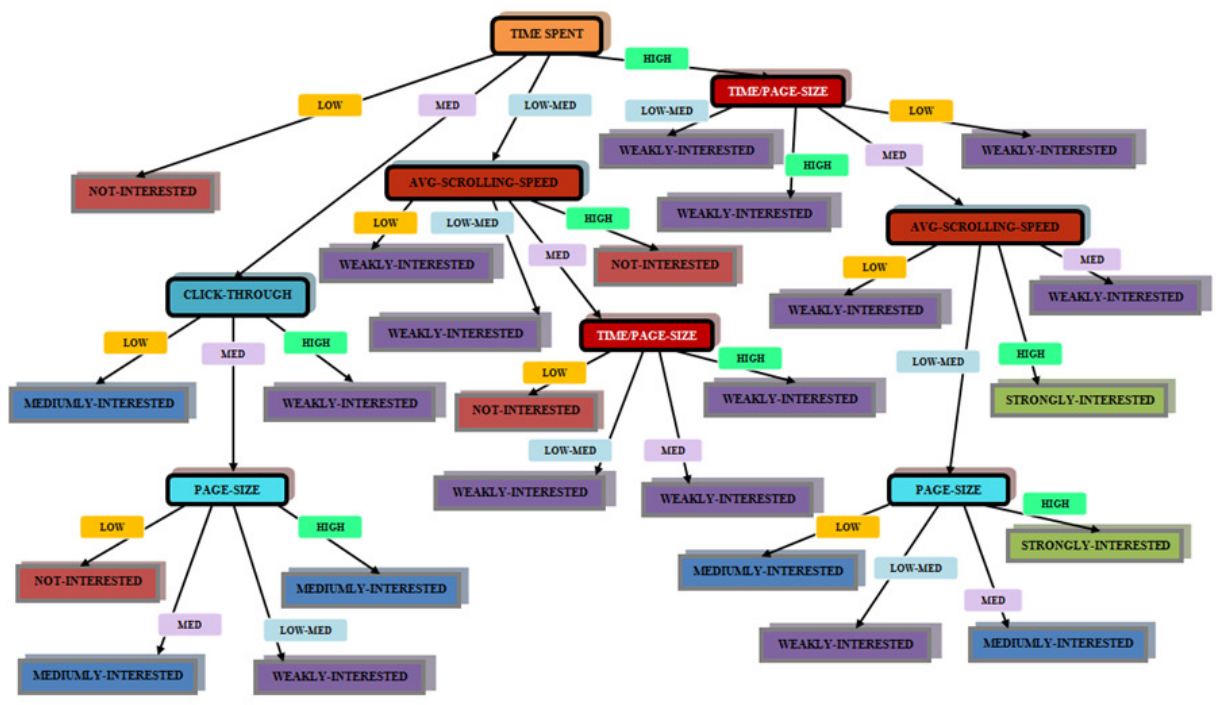

Figure 6. Decision tree for user interest classification

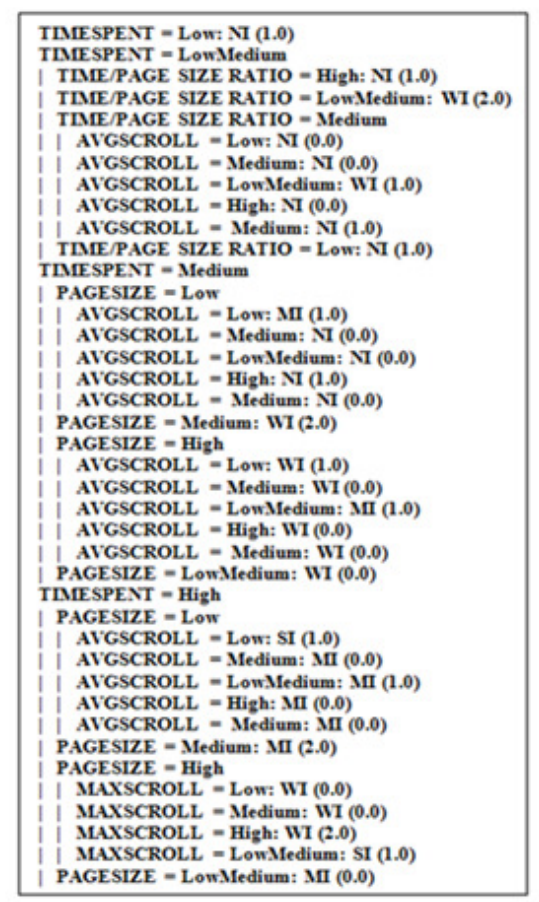

Figure 7. C4.5 algorithm generated tree equivalent rules

Table 10. Different query types of the single user in three sessions mapped to ODP categories

\begin{tabular}{|c|c|l|l|}
\hline $\begin{array}{c}\text { Session } \\
\text { no }\end{array}$ & $\begin{array}{c}\text { Query } \\
\text { type }\end{array}$ & \multicolumn{1}{|c|}{ Original query } & \multicolumn{1}{c|}{ Odp taxanomy category } \\
\hline 1 & Q1 & about data mining & data_mining/databases/software/computers \\
\hline 1 & Q2 & $\begin{array}{l}\text { about Web AND data } \\
\text { mining }\end{array}$ & data_mining/databases/software/computers \\
\hline 1 & Q3 & "Web and data mining" & data_mining/databases/software/computers \\
\hline 1 & Q4 & Introduction about & data_mining/databases/software/computers \\
\hline
\end{tabular}


International Journal on Cybernetics \& Informatics (IJCI) Vol. 3, No. 3, June 2014

\begin{tabular}{|c|c|l|l|}
\hline & & "Web and data mining" & \\
\hline 1 & Q5 & $\begin{array}{l}\text { definition of Web } \\
\text { mining }\end{array}$ & data_mining/databases/software/computers \\
\hline 2 & Q1 & about Sachin & $\begin{array}{l}\text { tendulkar,_sachin/players/india/full_memb } \\
\text { ers/icc/cricket/sports }\end{array}$ \\
\hline 2 & Q2 & $\begin{array}{l}\text { about Sachin AND his } \\
\text { records }\end{array}$ & $\begin{array}{l}\text { tendulkar,_sachin/players/india/full_memb } \\
\text { ers/icc/cricket/sports }\end{array}$ \\
\hline 2 & Q4 & $\begin{array}{l}\text { records of “Sachin } \\
\text { Tendulkar" }\end{array}$ & $\begin{array}{l}\text { tendulkar,_sachin/players/india/full_memb } \\
\text { ers/icc/cricket/sports }\end{array}$ \\
\hline 2 & Q5 & $\begin{array}{l}\text { number of centuries hit_cricket/sports } \\
\text { by Sachin }\end{array}$ & $\begin{array}{l}\text { tendulkar,_sachin/players/india/full_memb } \\
\text { ers/icc/cricket/sports }\end{array}$ \\
\hline 3 & Q1 & $\begin{array}{l}\text { University of madras } \\
\text { a/colleges_and_universities/education/refer } \\
\text { ence }\end{array}$ \\
\hline 3 & Q2 & $\begin{array}{l}\text { University of madras } \\
\text { AND distance } \\
\text { education }\end{array}$ & $\begin{array}{l}\text { university_of_madras/tamil_nadu/india/asi } \\
\text { a/colleges_and_universities/education/refer } \\
\text { ence }\end{array}$ \\
\hline 3 & Q3 & $\begin{array}{l}\text { "university of madras } \\
\text { and its distance } \\
\text { education scheme" }\end{array}$ & $\begin{array}{l}\text { university_of_madras/tamil_nadu/india/asi } \\
\text { a/colleges_and_universities/education/refer } \\
\text { ence }\end{array}$ \\
\hline Q4 & $\begin{array}{l}\text { About “university of } \\
\text { madras and its distance } \\
\text { mode education" }\end{array}$ & $\begin{array}{l}\text { university_of_madras/tamil_nadu/india/asi } \\
\text { a/colleges_and_universities/education/refer } \\
\text { ence }\end{array}$ \\
\hline $\begin{array}{l}\text { List of courses in } \\
\text { university of madras }\end{array}$ & $\begin{array}{l}\text { university_of_madras/tamil_nadu/india/asi } \\
\text { a/colleges_and_universities/education/refer } \\
\text { ence }\end{array}$ \\
\hline
\end{tabular}

\subsection{User Grouping}

After the estimation of categories, the proposed fuzzy model approaches fuzzy based classification on the specific query based pages which is referred by the user. Also this model generates the user grouping based on class labels assigned to each page (say, Not-Interest, WeakInterest, Medium-Interest, and Strong-Interest). Using this model we have examined 10 users and their' accessed 524 pages and performed both interest based classification as well as clustering based on class labels. Also we performed other types of grouping using user queries and related pages as well as categorization based grouping.

In order to simplify the grouping process single user visited 58 pages are processed and grouped according to its interest class labels as well as other groupings. The entire pages have belonged to any one of the class label. Each page is represented as separate edge and there exists a link between the corresponding classes it belongs to. Similarly all other groups are formed and represented as a graph and shown in Figure 8. In order to make query based user accessed page grouping, number of queries thrown by the user is counted and each query related pages are estimated using similarity measure. The following sets represent the number of queries and its related pages accessed by the single user along with the category in which each query belongs to (refer Figure 9). 


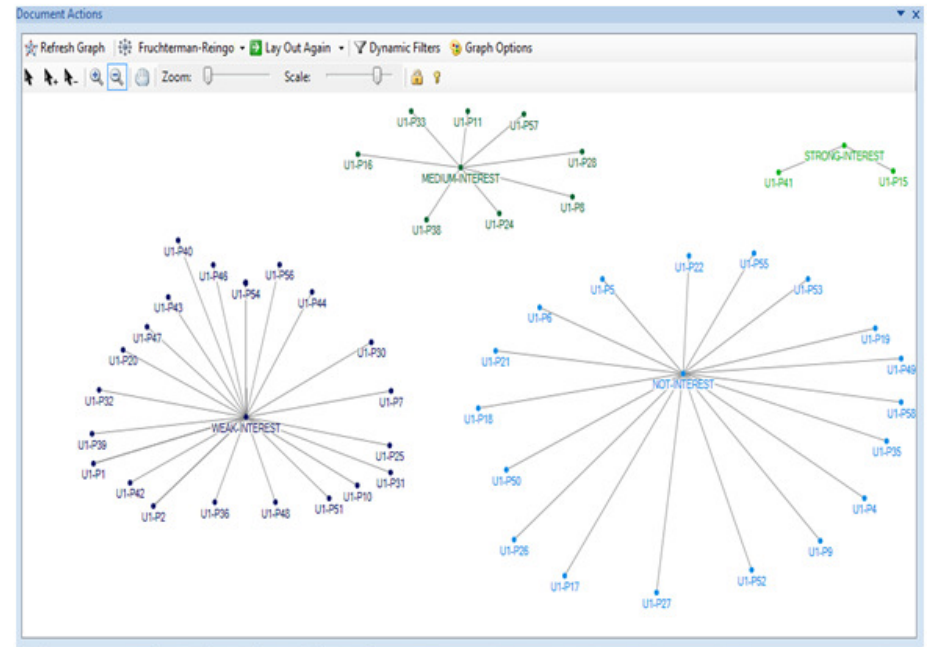

Figure 8. User grouping based on interest class labels

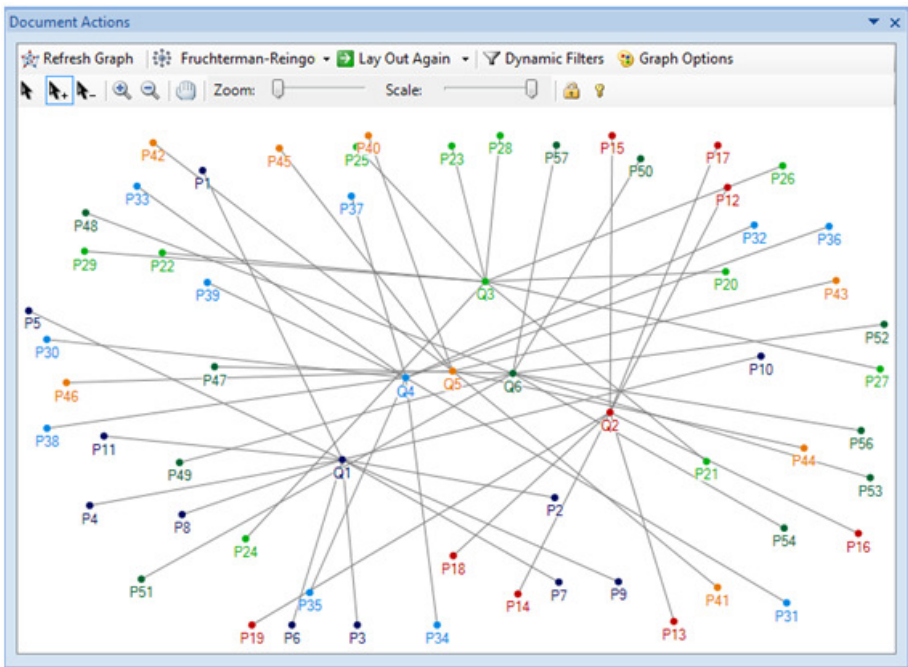

Figure 9. User query based Web page grouping

$$
\begin{aligned}
& \mathrm{Q}_{1}=\left\{\mathrm{P}_{1}, \mathrm{P}_{2}, \mathrm{P}_{3}, \mathrm{P}_{4}, \mathrm{P}_{5}, \mathrm{P}_{6}, \mathrm{P}_{7}, \mathrm{P}_{8}, \mathrm{P}_{9}, \mathrm{P}_{10}, \mathrm{P}_{11}\right\} \\
& \mathrm{Q}_{2}=\left\{\mathrm{P}_{12}, \mathrm{P}_{13}, \mathrm{P}_{14}, \mathrm{P}_{15}, \mathrm{P}_{16}, \mathrm{P}_{17}, \mathrm{P}_{18}, \mathrm{P}_{19}\right\} \\
& \mathrm{Q}_{3}=\left\{\mathrm{P}_{20}, \mathrm{P}_{21}, \mathrm{P}_{22}, \mathrm{P}_{23}, \mathrm{P}_{24}, \mathrm{P}_{25}, \mathrm{P}_{26}, \mathrm{P}_{27}, \mathrm{P}_{28}, \mathrm{P}_{29}\right\} \\
& \mathrm{Q}_{4}=\left\{\mathrm{P}_{30}, \mathrm{P}_{31}, \mathrm{P}_{32}, \mathrm{P}_{33}, \mathrm{P}_{34}, \mathrm{P}_{35}, \mathrm{P}_{36}, \mathrm{P}_{37}, \mathrm{P}_{38}, \mathrm{P}_{39}\right\} \\
& \mathrm{Q}_{5}=\left\{\mathrm{P}_{40}, \mathrm{P}_{41}, \mathrm{P}_{42}, \mathrm{P}_{43}, \mathrm{P}_{44}, \mathrm{P}_{45}, \mathrm{P}_{46}\right\} \\
& \mathrm{Q}_{6}=\left\{\mathrm{P}_{47}, \mathrm{P}_{48}, \mathrm{P}_{49}, \mathrm{P}_{50}, \mathrm{P}_{51}, \mathrm{P}_{52}, \mathrm{P}_{53}, \mathrm{P}_{54}, \mathrm{P}_{56}, \mathrm{P}_{57}\right\}
\end{aligned}
$$

The above sets represent six different queries thrown by asingle user (USER_1) during the entire search session. It also provides number of pages visited for each query and its corresponding page numbers from $\mathrm{P}_{1}$ to $\mathrm{P}_{58}$ are grouped.

$$
\begin{aligned}
& \mathrm{Q}_{1}=\left\{\mathrm{C}_{1}, \mathrm{C}_{2}\right\} ; \mathrm{Q}_{2}=\left\{\mathrm{C}_{1}, \mathrm{C}_{3}\right\} ; \mathrm{Q}_{3}=\left\{\mathrm{C}_{1}, \mathrm{C}_{2}\right\} ; \\
& \mathrm{Q}_{4}=\left\{\mathrm{C}_{1}, \mathrm{C}_{4}\right\} ; \mathrm{Q}_{5}=\left\{\mathrm{C}_{1}, \mathrm{C}_{2}, \mathrm{C}_{5}\right\} ; \mathrm{Q}_{6}=\left\{\mathrm{C}_{6}\right\}
\end{aligned}
$$

Similarly six different queries from $\mathrm{Q}_{1}-\mathrm{Q}_{6}$ are grouped based on the category label $\mathrm{C}_{1}-\mathrm{C}_{6}$. i.e. $\mathrm{Q}_{1}$, $\mathrm{Q}_{3}$ and $\mathrm{Q}_{5}$ belongs to category $\mathrm{C}_{2}$ ("web_mining/data_mining/databases/software/computers") which is shown in Figure 10. This query category is performed using ODP taxonomy as well as 
cosine similarity measure approach. Also each query related information and its relevant category label names are shown. Finally this model provides various relationships between the queries, categories and query related Web pages as well as their categories based on their similarities.

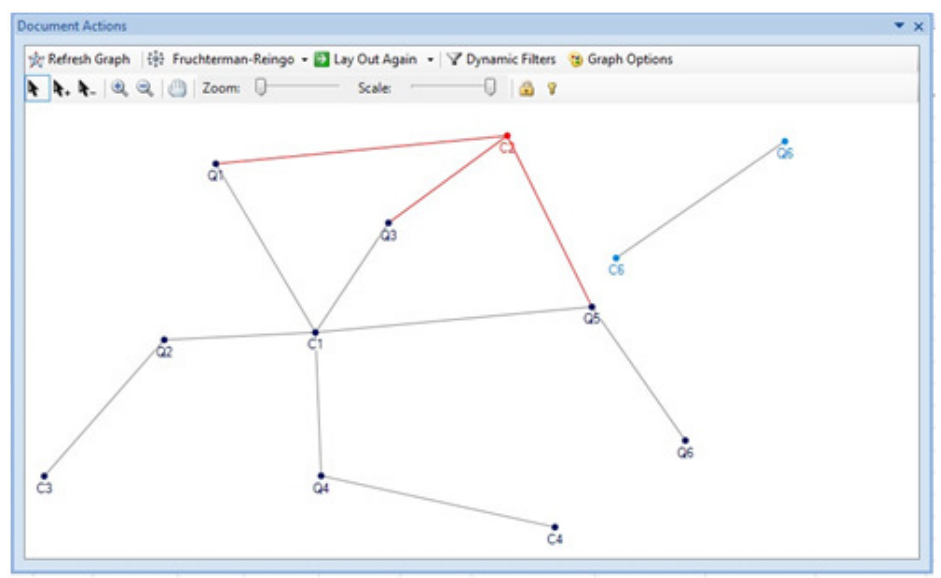

Figure 10. User query based Web page categorisation

$\mathrm{C}_{1}=$ "data_mining/databases/software/computers"

$\mathrm{C}_{2}=$ "web_mining/data_mining/databases/software/computers"

$\mathrm{C}_{3}=$ web_content_mining/web_mining/data_mining/databases/software/computers"

$\mathrm{C}_{4}=$ "books/object-oriented/software/computers"

$\mathrm{C}_{5}=$ "social_networking/web_mining/data_mining/databases/software/computers"

$\mathrm{C}_{6}=$ "tendulkar,_sachin/players/india/full_members/icc/cricket/sports"

$\mathrm{Q}_{1}$ - about data mining; $\mathrm{Q}_{2}$ - definition of Web mining

$\mathrm{Q}_{3}$-about Web AND data mining; $\mathrm{Q}_{4}$-Introduction about "Web and data mining

$\mathrm{Q}_{5^{-}}$"Web and data mining"; $\mathrm{Q}_{6^{-}}$records of "Sachin Tendulkar"

\section{DISCUSSION}

Personalized Web search depends greatly on the user data. Hence data collection becomes an integral part of any personalized Web search research. In order to achieve the proper collection of user search data, a specialized Web browser is utilised [5] and incorporated with the user model. This browser [5] has the ability to collect both the explicit as well as implicit data from the Web user. In particular this browser tracks user Scrolling Speed and other additional parameters such as: Page Size, Click-Through.

\subsection{Analysis of correlation model for user parameters}

The user search parameters are Time-spent, Scrolling-Speed, Click-through, Page-size, Maxscroll, Min-scroll and Time per Page-size ratio. Among these, the most significant parameters are Time-spent, Average-scrolling speed and Number of queries and also their relationship as well as significance are examined using Pearson correlation with 524 users' records. Time-spent and Average-scrolling speed are inversely proportional, Average-scrolling speed and number of queries are also inversely proportional and the number of query and Time-spent is directly proportional. In similar manner the other combinations of search parameter also estimated. The Pearson correlation is best suitable for measuring the inversely proportional relationship whereas Spearman Rank-Order Correlation and Kendall's tau-b are suitable for estimating the directly proportional relationship between the other user's parameters. 


\subsection{Z-Sample Test based result analysis}

Hypothesis test on user data based on time-spent on a Web page is considered based on statistical measures (mean, median and mode, etc) such as $\mathrm{H}_{0}$ and $\mathrm{H}_{1}$ called Null hypothesis and Alternative hypothesis respectively.

$\mathbf{H}_{\mathbf{0}}$ : A page is interest to a user if time-spent $>=15$ seconds and

$\mathbf{H}_{1}$ : A page is of not-interest to a user if time-spent $<15$ seconds.

Based on the above test results, the finalized user's minimum time-spent value will be 15 seconds. It also predicts that if it is greater than 15 seconds, the user is interested on the particular page; otherwise the user is not interested. This resultant value is tested by set of 10 user's data as shown in Figure 11. Similarly average scrolling speeds as well as other parameters like maximum-scrolling speed, minimum scrolling-speed, etc. are initialized using various statistical measures.

\subsection{Weighed N-Gram Based User Content Categorization}

Here the documents are represented by $\mathrm{N}$-gram frequency profiles. It is set of $\mathrm{N}$-grams arranged based on their frequency of that particular document. The grams are ordered according to their frequency values, in the form of chronological order. Table 11 provides single session query based user content categorization using $\mathrm{N}$-gram approach with the help of ODP Taxonomy.

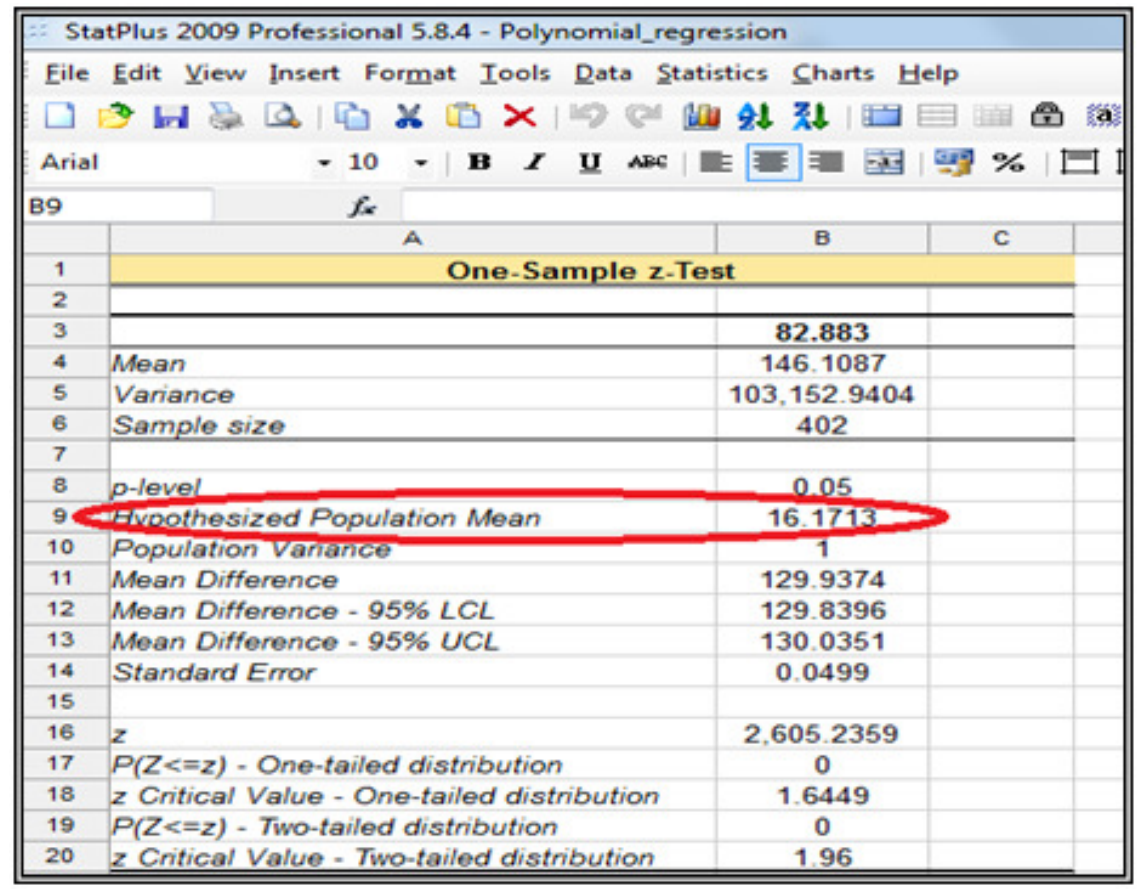

Figure 11.Snapshot representation of one-sample z-test result of user data 
International Journal on Cybernetics \& Informatics (IJCI) Vol. 3, No. 3, June 2014

Table 11. Weighed N-gram based categorization of session-1 user content

\begin{tabular}{|c|c|c|c|c|c|c|}
\hline $\begin{array}{l}\text { Sl. } \\
\text { no }\end{array}$ & $\begin{array}{l}\text { Gram } \\
\text { type }\end{array}$ & Sample & \begin{tabular}{|l|}
$\begin{array}{l}\text { Frequency } \\
\text { count }\end{array}$ \\
\end{tabular} & Category & $\begin{array}{l}\text { Frequency } \\
\text { count }\end{array}$ & $\begin{array}{l}\text { Similarity } \\
\text { measure }\end{array}$ \\
\hline \multirow[t]{4}{*}{1} & Trigram & $\begin{array}{l}\text { about Web } \\
\text { mining }\end{array}$ & 6 & $\begin{array}{l}\text { data_mining/databases/soft } \\
\text { ware/computers }\end{array}$ & 225 & 0.5814 \\
\hline & & $\begin{array}{l}\text { discover } \\
\text { patterns }\end{array}$ & 3 & $\begin{array}{l}\text { data_mining/databases/soft } \\
\text { ware/computers }\end{array}$ & 225 & 0.5814 \\
\hline & & $\begin{array}{l}\text { data mining } \\
\text { techniques }\end{array}$ & 3 & $\begin{array}{l}\text { data mining/reuse/software } \\
\text { engineering/software/com } \\
\text { puters }\end{array}$ & 16 & 0.5643 \\
\hline & & $\begin{array}{l}\text { data mining } \\
\text { methodolog } \\
\text { es }\end{array}$ & 2 & $\begin{array}{l}\text { data mining/reuse/software } \\
\text { engineering/software/com } \\
\text { puters }\end{array}$ & 16 & 0.5643 \\
\hline \multirow[t]{3}{*}{2} & bigram & Web mining & 24 & $\begin{array}{l}\text { data mining/databases/soft } \\
\text { ware/computers }\end{array}$ & 225 & 0.5814 \\
\hline & & about Web & 6 & $\begin{array}{l}\text { online publications/data m } \\
\text { ining/databases/software/co } \\
\text { mputers }\end{array}$ & 18 & 0.5221 \\
\hline & & data mining & 5 & $\begin{array}{l}\text { data_mining/databases/soft } \\
\text { ware/computers }\end{array}$ & 225 & 0.5814 \\
\hline \multirow[t]{3}{*}{3} & unigram & Web & 71 & $\begin{array}{l}\text { Web/servers/internet/softw } \\
\text { are/computers }\end{array}$ & 27 & 0.4872 \\
\hline & & Data & 16 & $\begin{array}{l}\text { 2008/events/data mining/d } \\
\text { atabases/software/computer } \\
\text { s }\end{array}$ & 20 & 0.4652 \\
\hline & & Mining & 63 & $\begin{array}{l}\text { drilling/tools and equipme } \\
\text { nt/mining, and drilling/busi } \\
\text { ness }\end{array}$ & 62 & 0.4361 \\
\hline
\end{tabular}

Here the $\mathrm{N}$ - value is restricted up to 3 (i.e., 1, 2 and 3). The $\mathrm{N}$-value is obtained by several experiments. Each gram from the specific N-value based ordered list is compared with ODP taxonomy and matched categories are chosen and listed based on their frequencies and ranked. Finally top ranked category is assigned as resultant category based on the contents referred during their search process.

\subsection{Fuzzification Model Selection}

This user model analyzes the distribution of various user parameters from the user data and compares it with various fuzzification membership functions such as triangle and trapezoidal fuzzy membership function based graphs. The best suitable fuzzification membership is chosen for user parameters based on the resemblance with the data distribution. This may be the reason for the resemblance of the entire user parameters into either Triangular or Trapezoidal fuzzification functions. And the model is not approached from the server side collected data set. Further, this model will enhance with inclusion of other fuzzifier models for doing fuzzification on user parameters.

\subsection{User Interest classification using fuzzy approach}

This model considered a set of four interest class labels I, and the expert members defined a set of parameters and constraints $P$ relevant to a particular topical user interest class. $I=\left\{i_{1}, i_{2}, i_{3}, i_{4}\right\}$ where $i_{1}, i_{2}, i_{3}, i_{4}$ are representing the four topical interest class label under consideration. $P=\left\{p_{1}\right.$, $\left.\mathrm{p}_{2}, \mathrm{p}_{3} \ldots \ldots \ldots \mathrm{p}_{\mathrm{m}}\right\}$ where $\mathrm{p}_{1}, \mathrm{p}_{2}, \mathrm{p}_{3} \ldots \ldots \ldots \mathrm{p}_{\mathrm{m}}$ represents the parameters and constraints of a particular topical interest class of user.

This research work makes a mild attempt to implement the concept of fuzzy rule based user model that integrates fuzzy techniques in reducing the complexity of identification of users' 
interest based on their log information. This user model developed and incorporated fuzzy rules for estimating user's interest class.

Table 12. Fuzzy rule base for user interest classification of USER_1

\begin{tabular}{|c|c|c|c|c|c|c|c|c|c|}
\hline $\begin{array}{c}\text { Page } \\
\text { no }\end{array}$ & $\begin{array}{c}\text { Rule } \\
\text { no }\end{array}$ & $\begin{array}{c}\text { Time } \\
\text { spent }\end{array}$ & $\begin{array}{c}\text { Avg } \\
\text { scroll }\end{array}$ & $\begin{array}{c}\text { Click } \\
\text { through }\end{array}$ & $\begin{array}{c}\text { Page } \\
\text { size }\end{array}$ & $\begin{array}{c}\text { Max } \\
\text { scroll }\end{array}$ & $\begin{array}{c}\text { Min } \\
\text { scroll }\end{array}$ & $\begin{array}{c}\text { Time/ } \\
\text { Page size ratio }\end{array}$ & Interest \\
\hline $\mathrm{P}_{1}$ & 2 & LMD & LOW & LOW & LOW & LOW & LOW & LMD & WI \\
\hline $\mathrm{P}_{2}$ & 12 & LMD & LMD & MED & LOW & MED & MED & MED & WI \\
\hline $\mathrm{P}_{3}$ & 5 & LMD & MED & MED & MED & MED & LOW & LMD & WI \\
\hline $\mathrm{P}_{4}$ & 10 & LMD & HGH & MED & LMD & MED & LOW & HGH & NI \\
\hline $\mathrm{P}_{5}$ & 1 & LOW & LOW & LOW & LOW & LOW & LOW & HGH & NI \\
\hline $\mathrm{P}_{6}$ & 1 & LOW & LOW & LOW & LOW & LOW & LOW & HGH & NI \\
\hline $\mathrm{P}_{7}$ & 20 & HGH & MED & LOW & HGH & HGH & LOW & LMD & WI \\
\hline $\mathrm{P}_{8}$ & 3 & MED & LOW & LOW & LOW & LOW & LOW & MED & MI \\
\hline $\mathrm{P}_{9}$ & 1 & LOW & LOW & LOW & LOW & LOW & LOW & HGH & NI \\
\hline $\mathrm{P}_{10}$ & 2 & LMD & LOW & LOW & LOW & LOW & LOW & LMD & WI \\
\hline $\mathrm{P}_{11}$ & 6 & MED & LMD & MED & HGH & MED & MED & LMD & MI \\
\hline $\mathrm{P}_{12}$ & 20 & HGH & MED & LOW & HGH & HGH & LOW & LMD & WI \\
\hline $\mathrm{P}_{13}$ & 2 & LMD & LOW & LOW & LOW & LOW & LOW & LMD & WI \\
\hline $\mathrm{P}_{14}$ & 2 & LMD & LOW & LOW & LOW & LOW & LOW & LMD & WI \\
\hline $\mathrm{P}_{15}$ & 4 & HGH & LOW & LOW & LOW & LOW & LOW & HGH & SI \\
\hline $\mathrm{P}_{16}$ & 13 & MED & LMD & MED & MED & MED & MED & LOW & MI \\
\hline $\mathrm{P}_{17}$ & 18 & LMD & MED & LOW & LOW & MED & MED & MED & NI \\
\hline $\mathrm{P}_{18}$ & 16 & LMD & MED & LOW & LOW & MED & LOW & LOW & NI \\
\hline $\mathrm{P}_{19}$ & 1 & LOW & LOW & LOW & LOW & LOW & LOW & HGH & NI \\
\hline $\mathrm{P}_{20}$ & 2 & LMD & LOW & LOW & LOW & LOW & LOW & LMD & WI \\
\hline
\end{tabular}

This model tested 524 records from 10 different users and assigned class labels using fuzzy approach. It also performed filtering of the Not-interest and Weak-Interest pages and recommended only Medium as well Strong-Interest pages. Finally it performed categorization of the users based on their class labels. Here the class labels are represented by numerical values such as: $1,2,3$, and 4 . the corresponding linguistic labels of each user search parameters are represented in the Table 12.

Table 13. Classification of user interest with Category labels

\begin{tabular}{|c|c|l|l|}
\hline PAGE_NO & USER_NAME & $\begin{array}{c}\text { CATEGORY } \\
\text { (DOMAIN) }\end{array}$ & $\begin{array}{l}\text { INTEREST } \\
\text { CLASS LABEL }\end{array}$ \\
\hline $\mathbf{P}_{\mathbf{1}}$ & USER_1 & $\begin{array}{l}\text { Web_Mining } \\
\text { (Computers) }\end{array}$ & $\begin{array}{l}\text { WEAKLY- } \\
\text { INTERESTED }\end{array}$ \\
\hline $\mathbf{P}_{\mathbf{2}}$ & USER_1 & $\begin{array}{l}\text { Web_Mining } \\
\text { (Computers) }\end{array}$ & $\begin{array}{l}\text { WEAKLY- } \\
\text { INTERESTED }\end{array}$ \\
\hline $\mathbf{P}_{\mathbf{3}}$ & USER_1 & $\begin{array}{l}\text { Web_Mining } \\
\text { (Computers) }\end{array}$ & $\begin{array}{l}\text { WEAKLY- } \\
\text { INTERESTED }\end{array}$ \\
\hline $\mathbf{P}_{\mathbf{4}}$ & USER_1 & $\begin{array}{l}\text { Web_Mining } \\
\text { (Computers) }\end{array}$ & $\begin{array}{l}\text { NOT- } \\
\text { INTERESTED }\end{array}$ \\
\hline $\mathbf{P}_{\mathbf{5}}$ & USER_1 & $\begin{array}{l}\text { Web_Mining } \\
\text { (Computers) }\end{array}$ & $\begin{array}{l}\text { NOT- } \\
\text { INTERESTED }\end{array}$ \\
\hline $\mathbf{P}_{\mathbf{6}}$ & USER_1 & $\begin{array}{l}\text { Web_Mining } \\
\text { (Computers) }\end{array}$ & $\begin{array}{l}\text { NOT- } \\
\text { INTERESTED }\end{array}$ \\
\hline $\mathbf{P}_{\mathbf{7}}$ & USER_1 & $\begin{array}{l}\text { data_mining } \\
\text { (Computers) }\end{array}$ & $\begin{array}{l}\text { WEAKLY- } \\
\text { INTERESTED }\end{array}$ \\
\hline $\mathbf{P}_{\mathbf{8}}$ & USER_1 & $\begin{array}{l}\text { data_mining } \\
\text { (Computers) }\end{array}$ & $\begin{array}{l}\text { MEDIUMLY- } \\
\text { INTERESTED }\end{array}$ \\
\hline $\mathbf{P}_{\mathbf{9}}$ & USER_1 & Web_Mining & NOT- \\
\hline
\end{tabular}




\begin{tabular}{|c|c|l|l|}
\hline & & (Computers) & INTERESTED \\
\hline $\mathbf{P}_{\mathbf{1 0}}$ & USER_1 & $\begin{array}{l}\text { Web_Mining } \\
\text { (Computers) }\end{array}$ & $\begin{array}{l}\text { WEAKLY- } \\
\text { INTERESTED }\end{array}$ \\
\hline $\mathbf{P}_{\mathbf{1 1}}$ & USER_1 & $\begin{array}{l}\text { Web_Mining } \\
\text { (Computers) }\end{array}$ & $\begin{array}{l}\text { MEDIUMLY- } \\
\text { INTERESTED }\end{array}$ \\
\hline $\mathbf{P}_{\mathbf{1 2}}$ & USER_1 & $\begin{array}{l}\text { Web_Mining } \\
\text { (Computers) }\end{array}$ & $\begin{array}{l}\text { WEAKLY- } \\
\text { INTERESTED }\end{array}$ \\
\hline $\mathbf{P}_{\mathbf{1 3}}$ & USER_1 & $\begin{array}{l}\text { Web_Mining } \\
\text { (Computers) }\end{array}$ & $\begin{array}{l}\text { WEAKLY- } \\
\text { INTERESTED }\end{array}$ \\
\hline $\mathbf{P}_{\mathbf{1 4}}$ & USER_1 & $\begin{array}{l}\text { Web_Mining } \\
\text { (Computers) }\end{array}$ & $\begin{array}{l}\text { WEAKLY- } \\
\text { INTERESTED }\end{array}$ \\
\hline $\mathbf{P}_{\mathbf{1 5}}$ & USER_1 & $\begin{array}{l}\text { Web_Mining } \\
\text { (Computers) }\end{array}$ & $\begin{array}{l}\text { STRONGLY- } \\
\text { INTERESTED }\end{array}$ \\
\hline $\mathbf{P}_{\mathbf{1 6}}$ & USER_1 & $\begin{array}{l}\text { Web_Mining } \\
\text { (Computers) }\end{array}$ & $\begin{array}{l}\text { MEDIUMLY- } \\
\text { INTERESTED }\end{array}$ \\
\hline $\mathbf{P}_{\mathbf{1 7}}$ & USER_1 & $\begin{array}{l}\text { Web_Content_Mining } \\
\text { (Computers) }\end{array}$ & $\begin{array}{l}\text { NOT- } \\
\text { INTERESTED }\end{array}$ \\
\hline $\mathbf{P}_{\mathbf{1 0}}$ & USER_1 & $\begin{array}{l}\text { data_mining } \\
\text { (Computers) }\end{array}$ & $\begin{array}{l}\text { NOT- } \\
\text { INTERESTED }\end{array}$ \\
\hline $\mathbf{P}_{\mathbf{1 9}}$ & USER_1 & $\begin{array}{l}\text { data_mining } \\
\text { (Computers) }\end{array}$ & $\begin{array}{l}\text { NOT- } \\
\text { INTERESTED }\end{array}$ \\
\hline $\mathbf{P}_{\mathbf{2 0}}$ & USER_1 & $\begin{array}{l}\text { data_mining } \\
\text { (Computers) }\end{array}$ & $\begin{array}{l}\text { WEAKLY- } \\
\text { INTERESTED }\end{array}$ \\
\hline
\end{tabular}

Here NI, WI, MI, and SI represent Not-Interested, Weakly-Interested, Mediumly-Interested, and Strongly-Interested respectively. Similarly LOW, LMD, MED, and HGH are indicated as Low, Low-Medium, Medium, and High respectively. Table 13 provide the single user accessed 20 pages, its category labels and their corresponding interest class labels. Category label consists of both main (root) category represented within the bracket as well as its sub (ancestor) category of the page which belongs to is shown. i.e. USER_1 accessed Page No: 1 belongs to Main category: Computer and sub-category: Web_Mining.

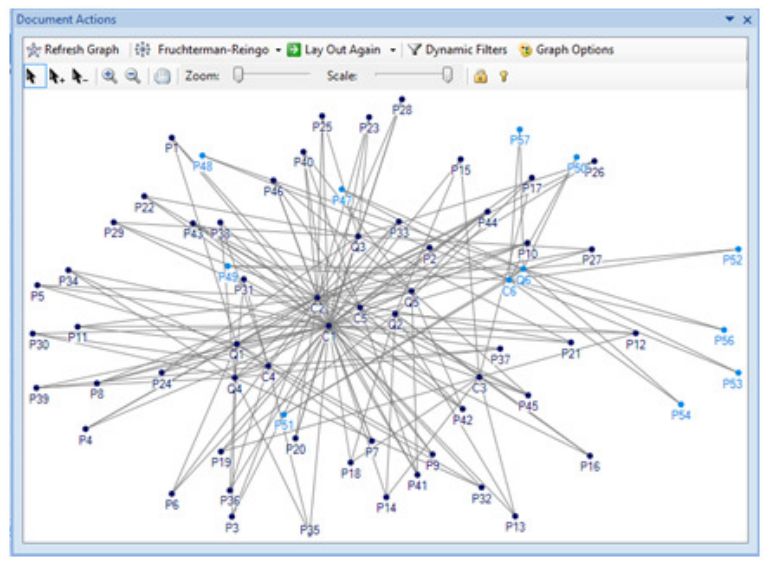

Figure 12. Category based USER_1 accessed Web pages and its query grouping 


\subsection{Grouping the User Web pages and Queries based on Categories}

This model also performs the grouping of user accessed pages and its related queries based on their categories. This approach provides the inference of the users' major interest area in order to provide their anticipated based on their interest. Here the USER_1 accessed 58 Web pages are grouped and the result is shown in Figure 12. This user accessed Web pages mostly belonged to $\mathrm{C}_{1}$. So it infers that the USER_1 is interested in "data_mining/databases/software/computers" category based contents shown in Figure 13.

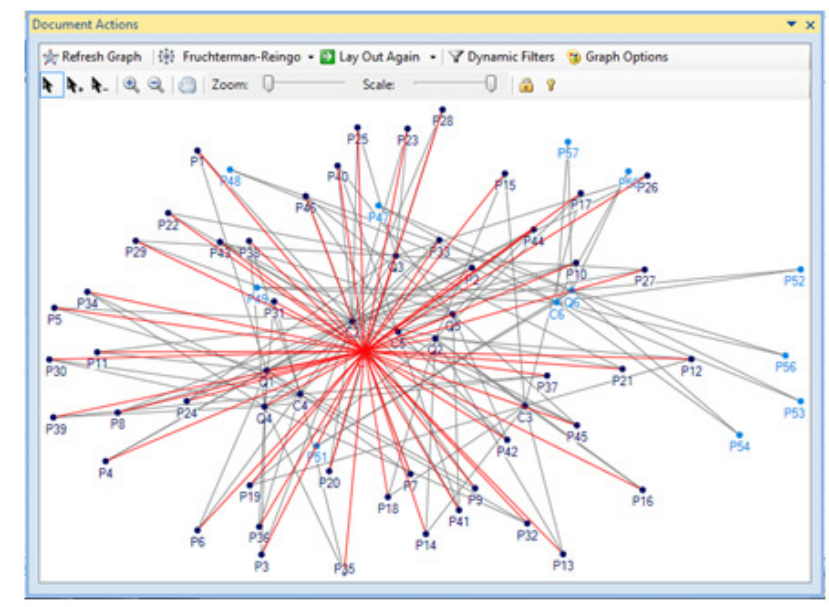

Figure 13. Category $\mathrm{C}_{1}$ of USER_1 accessed Web pages and its query grouping

Table 14. Various Performance measures of Bayesian and Fuzzy Classifier

\begin{tabular}{|c|c|c|c|c|c|c|}
\hline $\begin{array}{c}\text { Sl. } \\
\text { No }\end{array}$ & $\begin{array}{c}\text { Method } \\
\text { Approached }\end{array}$ & $\begin{array}{c}\text { Total } \\
\text { no. of } \\
\text { records }\end{array}$ & $\begin{array}{c}\text { No. of } \\
\text { Correct } \\
\text { classification }\end{array}$ & $\begin{array}{c}\text { No. of In- } \\
\text { Correct } \\
\text { classification }\end{array}$ & $\begin{array}{c}\text { Successful } \\
\text { classification } \\
\text { rate }\end{array}$ & $\begin{array}{c}\text { Failure } \\
\text { classification } \\
\text { rate }\end{array}$ \\
\hline 1 & $\begin{array}{c}\text { Bayesian } \\
\text { Classifier }\end{array}$ & 524 & 398 & 126 & $75.9541 \%$ & $24.0450 \%$ \\
\hline 2 & $\begin{array}{c}\text { Fuzzy } \\
\text { Classifier }\end{array}$ & 524 & 470 & 54 & $89.6946 \%$ & $10.3053 \%$ \\
\hline
\end{tabular}

In order to test the performance of this model, the Bayesian classifier is approached with the same data set (524 records from 10 different users). The results show that the Fuzzy rule based user model provides remarkable results than Bayesian classifier which is shown in Table 14. Compared with Bayesian classifier the proposed model provides less number of failure rate classifications and higher accuracy rate of successful classification.

\section{CONCLUSION AND FUTURE WORK}

The proposed User interest based classification in a personalized Web search using the fuzzy model delivered the acceptable rate of classification results. Heuristic based approach is incorporated in this model, so it enhances the accuracy of the classification of the user interest. The fuzzification functions are playing a major role for handling uncertainty data in such vague environment. Here the fuzzification is performed based on specific membership function and the selection of a specific membership function is based on the nature of the search data. Therefore caution has to be exercised during the membership function selection process. In future, the same model shall be drawn-out using Artificial Neural Networks (ANN). Genetic algorithm can be used for estimating errors and automatic fuzzifier selection process. In addition, the same user 
International Journal on Cybernetics \& Informatics (IJCI) Vol. 3, No. 3, June 2014

model will be extended to perform the prediction of user's interest fluctuation as well as interest decays.

\section{REFERENCES}

[1] Herrera, Mauro Rojas, Edleno Silva de Moura, Marco Cristo, Thomaz Philippe Silva, and Altigran Soares da Silva. "Exploring features for the automatic identification of user goals in web search." Information processing \& management 46, no. 2 (2010): 131-142.

[2] Yu, Jie, Jie Gong, and Fangfang Liu. "Generation of Semantic Interactive Environment for Personalized Search." In Computer and Information Technology (CIT), 2011 IEEE 11th International Conference on, pp. 443-448. IEEE, 2011.

[3] Kamalanathan, Selvakumar, and Sendhilkumar Selvaraju. "Collaborative Approaches for Personalized Web Search Using Fuzzy Neural Networks." InGlobal Trends in Information Systems and Software Applications, pp. 367-376. Springer Berlin Heidelberg, 2012.

[4] Delbru, Renaud, Stephane Campinas, and Giovanni Tummarello. "Searching web data: An entity retrieval and high-performance indexing model." Web Semantics: Science, Services and Agents on the World Wide Web 10 (2012): 33-58.

[5] Sendhilkumar, S., and T. V. Geetha. "Personalized ontology for web search personalization." In Proceedings of the 1st Bangalore annual Compute conference, p. 18. ACM, 2008.

[6] Dinh, Duy, and Lynda Tamine. "Towards a context sensitive approach to searching information based on domain specific knowledge sources." Web Semantics: Science, Services and Agents on the World Wide Web 12 (2012): 41-52.

[7] Kumar, AP Siva, P. Premchand, and A. Govardhan. "Query-based summarizer based on similarity of sentences and word frequency." International Journal of Data Mining \& Knowledge Management Process 1, no. 3 (2011).

[8] Lin, Gu-Li, Hong Peng, Qian-Li Ma, Jia Wei, and Jiang-Wei Qin. "A Keyword Based Prototype for Web Search Result Diversification." Journal of Information Science \& Engineering 28, no. 3 (2012).

[9] Chen, Tzung-Shi, Yen-Ssu Chou, and Tzung-Cheng Chen. "Mining user movement behavior patterns in a mobile service environment." Systems, Man and Cybernetics, Part A: Systems and Humans, IEEE Transactions on 42, no. 1 (2012): 87-101.

[10] Kim, Jin Young, Kevyn Collins-Thompson, Paul N. Bennett, and Susan T. Dumais. "Characterizing web content, user interests, and search behavior by reading level and topic." In Proceedings of the fifth ACM international conference on Web search and data mining, pp. 213-222. ACM, 2012.

[11] Rahul Verma, Kshitij Pathak, "Personalized Concept and Fuzzy Based User Profile from Search Engine Queries". International Journal of Emerging Technology and Advanced Engineering, Volume 2, Issue 12, December 2012.

[12] Velásquez, Juan D. "Web mining and privacy concerns: Some important legal issues to be consider before applying any data and information extraction technique in web-based environments." Expert Systems with Applications 40, no. 13 (2013): 5228-5239.

[13] Glorio, Octavio, Jose-Norberto Mazón, Irene Garrigós, and Juan Trujillo. "A personalization process for spatial data warehouse development." Decision Support Systems 52, no. 4 (2012): 884-898.

[14] Guerbas, Abdelghani, Omar Addam, Omar Zaarour, Mohamad Nagi, Ahmad Elhajj, Mick Ridley, and Reda Alhajj. "Effective web log mining and online navigational pattern prediction." KnowledgeBased Systems 49 (2013): 50-62.

[15] Marin, Lucas, David Isern, Antonio Moreno, and Aida Valls. "On-line dynamic adaptation of fuzzy preferences." Information Sciences 220 (2013): 5-21.

[16] Castellano, Giovanna, Anna Maria Fanelli, and Maria Alessandra Torsello. "NEWER: A system for NEuro-fuzzy WEb Recommendation." Applied Soft Computing 11, no. 1 (2011): 793-806.

[17] Ahmad, Mohd Wazih, and M. A. Ansari. "A Survey: Soft Computing in Intelligent Information Retrieval Systems." In Computational Science and Its Applications (ICCSA), 2012 12th International Conference on, pp. 26-34. IEEE, 2012.

[18] Zadeh, Lotfi A. "Fuzzy sets." Information and control 8, no. 3 (1965): 338-353.

[19] Zadeh, Lotfi A. "Fuzzy logic= computing with words." Fuzzy Systems, IEEE Transactions on 4, no. 2 (1996): 103-111.

[20] Verbiest, Nele, Chris Cornelis, and Francisco Herrera. "FRPS: A fuzzy rough prototype selection method." Pattern Recognition 46, no. 10 (2013): 2770-2782. 
[21] Brucker, Florian, Fernando Benites, and Elena Sapozhnikova. "Multi-label classification and extracting predicted class hierarchies." Pattern Recognition44, no. 3 (2011): 724-738.

[22] Arbelaitz, Olatz, Ibai Gurrutxaga, Aizea Lojo, Javier Muguerza, Jesús Maria Pérez, and Iñigo Perona. "Web usage and content mining to extract knowledge for modelling the users of the Bidasoa Turismo website and to adapt it." Expert Systems with Applications 40, no. 18 (2013): 7478-7491.

[23] Thilagu, M., and R. Nadarajan. "INVESTIGATING SIGNIFICANT CHANGES IN USERS'INTEREST ON WEB TRAVERSAL PATTERNS." International Journal on on Cybernetics \& Informatics ( IJCI) Vol.2, No.4, August 2013.

[24] Serrano-Guerrero, Jesus, Francisco P. Romero, and Jose A. Olivas. "Hiperion: A fuzzy approach for recommending educational activities based on the acquisition of competences." Information Sciences 248 (2013): 114-129.

[25] Li, Sheng-Tun, and Fu-Ching Tsai. "A fuzzy conceptualization model for text mining with application in opinion polarity classification." Knowledge-Based Systems 39 (2013): 23-33.

[26] Pal, Sankar K., Saroj K. Meher, and Soumitra Dutta. "Class-dependent rough-fuzzy granular space, dispersion index and classification." Pattern Recognition45, no. 7 (2012): 2690-2707.

[27] 26.a. Mansoori, E. G., M. J. Zolghadri, and S. D. Katebi. "Using distribution of data to enhance performance of fuzzy classification systems." Iranian Journal of Fuzzy Systems 4, no. 1 (2007): 2136.

[28] Ropero, Jorge, Ariel Gómez, Alejandro Carrasco, and Carlos León. "A Fuzzy Logic intelligent agent for Information Extraction: Introducing a new Fuzzy Logic-based term weighting scheme." Expert Systems with Applications 39, no. 4 (2012): 4567-4581.

[29] Kyoomarsi, F., H. Khosravi, E. Eslami, and M. Davoudi. "Extraction-based text summarization using fuzzy analysis." Iranian Journal of Fuzzy Systems 7, no. 3 (2010): 15-32.

[30] Li, Ming, Lu Liu, and Chuan-Bo Li. "An approach to expert recommendation based on fuzzy linguistic method and fuzzy text classification in knowledge management systems." Expert Systems with Applications 38, no. 7 (2011): 8586-8596.

[31] Siddiqui, Fasahat Ullah, Nor Ashidi Mat Isa, and Abid Yahya. "Outlier rejection fuzzy c-means (ORFCM) algorithm for image segmentation." Turkish Journal of Electrical Engineering \& Computer Sciences 21, no. 6 (2013).

[32] George J.Klir, Bo Yuan. Fuzzy Sets and Fuzzy Logic Theory and Applications. Prentice Hall P T R Upper Saddle River, New Jersey: Prentice Hall, 1995.

[33] Timothy J. Ross. Fuzzy Logic with Engineering Applications. 3rd Edition, University of New Mexico, USA: John Wiley \& Sons, Ltd, 2010.

[34] Qureshi, M. Rizwan Jameel, Mahaboob Sharief Shaik, and Nayyar Iqbal. "Using Fuzzy Logic to Evaluate Normalization Completeness for an Improved Database Design." International Journal of Information Technology and Computer Science (IJITCS) 4, no. 2 (2012): 48.

[35] Batra, Geetika, and Mahima Trivedi. "AFuzzy APPROACH FOR SOFTWARE EFFORT ESTIMATION." International Journal Cybernetics \& Informatics ( IJCI) Vol.2, No.1, February 2013.

[36] George J.Klir, Bo Yuan. Fuzzy Sets, Fuzzy Logic, and Fuzzy Systems. World Scientific Publishing Co Pte Ltd, Singapore: WSP, 1996.

[37] Ganesh M. Introduction to Fuzzy Sets and Fuzzy Logic. PHI Learning Pvt. Ltd: PHI, 2006.

[38] Bede and Barnabas. Mathematics of Fuzzy Sets and Fuzzy Logic. Studies in Fuzziness and Soft Computing: Springer, 2013.

[39] Ren, Yan, Xiaodong Liu, and Jiannong Cao. "A parsimony fuzzy rule-based classifier using axiomatic fuzzy set theory and support vector machines."Information Sciences 181, no. 23 (2011): 5180-5193.

[40] Loia, Vincenzo, and Sabrina Senatore. "A fuzzy-oriented sentic analysis to capture the human emotion in Web-based content." Knowledge-Based Systems(2013).

[41] Alcalá-Fdez, Jesús, Rafael Alcalá, María José Gacto, and Francisco Herrera. "Learning the membership function contexts for mining fuzzy association rules by using genetic algorithms." Fuzzy Sets and Systems 160, no. 7 (2009): 905-921.

[42] Weber, Siegfried. "A general concept of fuzzy connectives, negations and implications based on $<$ i $>$ $\mathrm{t}</ \mathrm{i}>$-norms and $<\mathrm{i}>\mathrm{t}</ \mathrm{i}>$-conorms." Fuzzy sets and systems 11, no. 1 (1983): 103-113.

[43] Srikant, Ramakrishnan, Sugato Basu, Ni Wang, and Daryl Pregibon. "User browsing models: relevance versus examination." In Proceedings of the 16th ACM SIGKDD international conference on Knowledge discovery and data mining, pp. 223-232. ACM, 2010.

[44] Zadeh, Lotfi A. "A fuzzy-set-theoretic interpretation of linguistic hedges." (1972): 4-34. 
[45] Samuel, O. W., M. O. Omisore, and B. A. Ojokoh. "A web based decision support system driven by fuzzy logic for the diagnosis of typhoid fever." Expert Systems with Applications 40, no. 10 (2013): 4164-4171.

[46] Djam, X. Y., G. M. Wajiga, Y. H. Kimbi, and N. V. Blamah. "A Fuzzy Expert System for the Management of Malaria." International Journal of Pure \& Applied Sciences \& Technology 5, no. 2 (2011).

[47] Chang, Robin LP, and Theodosios Pavlidis. "Fuzzy decision tree algorithms."Systems, Man and Cybernetics, IEEE Transactions on 7, no. 1 (1977): 28-35.

[48] Dong, Ming, and Ravi Kothari. "Look-ahead based fuzzy decision tree induction." Fuzzy Systems, IEEE Transactions on 9, no. 3 (2001): 461-468.

[49] Mitra, Sushmita, Kishori M. Konwar, and Sankar K. Pal. "Fuzzy decision tree, linguistic rules and fuzzy knowledge-based network: generation and evaluation."Systems, Man, and Cybernetics, Part C: Applications and Reviews, IEEE Transactions on 32, no. 4 (2002): 328-339.

[50] LEVASHENKO, Vitaly, and Penka MARTINCOVÁ. "Fuzzy Decision Tree for Parallel Processing Support." Journal of Information, Control and Management Systems 3, no. 1 (2005).

[51] MATIAŠKO, Karol, Ján BOHÁČIK, Vitaly LEVASHENKO, and Štefan KOVALÍK. "Learning fuzzy rules from fuzzy decision trees." Journal of Information, Control and Management Systems 4, no. 2 (2006).

[52] Pulkkinen, Pietari, and Hannu Koivisto. "Fuzzy classifier identification using decision tree and multiobjective evolutionary algorithms." International Journal of Approximate Reasoning 48, no. 2 (2008): 526-543.

[53] Abolfazl Kazemia, Elahe Mehrzadeganb. A New Algorithm for Optimization of Fuzzy Decision Tree in Data Mining. Journal of Optimization in Industrial Engineering 2011; 7: 29-35.

[54] Daveedu Raju Adidela, Jaya Suma. G, Lavanya Devi. G. Construction of Fuzzy Decision Tree using Expectation Maximization Algorithm. Journal of Computer Science and Management Research 2012; 1: 416-424. 